\title{
Investigation of the role of typhoid toxin in acute typhoid fever in a human challenge model
}

\author{
Malick M. Gibani ${ }^{1,2 \star}$, Elizabeth Jones ${ }^{1}$, Amber Barton', Celina Jin1', Juliette Meek1, Susana Camara1, \\ Ushma Galal ${ }^{3}$, Eva Heinz ${ }^{4,5}$, Yael Rosenberg-Hasson ${ }^{6}$, Gerlinde Obermoser ${ }^{6}$, Claire Jones ${ }^{1}$, \\ Danielle Campbell ${ }^{1}$, Charlotte Black', Helena Thomaides-Brears', Christopher Darlow', \\ Christina Dold', Laura Silva-Reyes', Luke Blackwell', Maria Lara-Tejero', Xuyao Jiao7, Gabrielle Stack7, \\ Christoph J. Blohmke', Jennifer Hill', Brian Angus $\mathbb{1}^{8}{ }^{8}$, Gordon Dougan ${ }^{4,9}$, Jorge Galán $\mathbb{D}^{7}$ and \\ Andrew J. Pollard'
}

\begin{abstract}
Salmonella Typhi is a human host-restricted pathogen that is responsible for typhoid fever in approximately 10.9 million people annually'. The typhoid toxin is postulated to have a central role in disease pathogenesis, the establishment of chronic infection and human host restriction ${ }^{2-6}$. However, its precise role in typhoid disease in humans is not fully defined. We studied the role of typhoid toxin in acute infection using a randomized, double-blind $S$. Typhi human challenge model ${ }^{7}$. Forty healthy volunteers were randomized (1:1) to oral challenge with $10^{4}$ colony-forming units of wild-type or an isogenic typhoid toxin deletion mutant (TN) of S. Typhi. We observed no significant difference in the rate of typhoid infection (fever $\geq 38^{\circ} \mathrm{C}$ for $\geq 12 \mathrm{~h}$ and/or $S$. Typhi bacteremia) between participants challenged with wild-type or TN S. Typhi (15 out of 21 (71\%) versus 15 out of $19(79 \%) ; P=0.58)$. The duration of bacteremia was significantly longer in participants challenged with the TN strain compared with wild-type (47.6 hours (28.9-97.0) versus 30.3(3.6-49.4); $P \leq 0.001)$. The clinical syndrome was otherwise indistinguishable between wild-type and TN groups. These data suggest that the typhoid toxin is not required for infection and the development of early typhoid fever symptoms within the context of a human challenge model. Further clinical data are required to assess the role of typhoid toxin in severe disease or the establishment of bacterial carriage.

Several preclinical studies have described the structure and function of the typhoid toxin in vitro and in small-animal models ${ }^{2-6}$. Systemic administration of typhoid toxin to C57BL/6 mice results in the reproduction of many characteristic symptoms of typhoid fever; other studies have suggested that typhoid toxin may contribute to the establishment of chronic infection ${ }^{4,5}$. It remains unclear how the surrogate end points of illness in mice-such as lethargy, weight loss, behavioral and motor changes ${ }^{3,8}$-are representative of acute typhoid fever in humans. The toxin is also encoded by $>40$ clade B non-typhoidal Salmonella (NTS) serovars that display a broad host range and a distinct clinical phenotype to $S$. Typhi and Paratyphi ${ }^{9-11}$,
\end{abstract}

although some typhoid toxin-expressing NTS serovars appear to cause an enteric fever-like syndrome ${ }^{12}$. Importantly, no previous studies have characterized the role of typhoid toxin in a human model of disease.

We aimed to characterize the role of typhoid toxin in human infection and pathogenesis using an $S$. Typhi human challenge model ${ }^{7}$. This model has previously been used to test novel liveattenuated (MO1ZH09) (ref. ${ }^{13}$ ) and Vi-conjugate (Typbar-TCV) typhoid vaccines ${ }^{14}$. We manufactured two challenge strains of $S$. Typhi to good manufacturing practice (GMP) standards. We used the wild-type S. Typhi Quailes strain (genotype 3.0.1 (ref. ${ }^{15}$ )) as the parent strain to generate an isogenic typhoid toxin-deficient knockout strain (TN), as described previously ${ }^{16}$. Whole-genome sequencing confirmed the absence of the typhoid toxin pathogenicity islet in the TN strain (Supplementary Information). The wild-type and TN strains harbored no other differences in relation to known key virulence factors (Supplementary Information). In particular, there were no differences identified in Salmonella pathogenicity island 7, a region encoding genes required for expression of the Vi-capsule-a key virulence factor in the pathogenesis of $S$. Typhi. Differences between strains were confined to highly variable regions encoding phage proteins, which were not known to impact on bacterial survival in the environment or persistence in the human host. However, deletion of the entire typhoid toxin pathogenicity island was associated with increased bacterial burden in a mouse model of $S$. Typhi infection, compared with a strain expressing a catalytic mutant of typhoid toxin ( $c d t B^{\mathrm{H} 160 \mathrm{Q}} p l t B^{\mathrm{S} 35 \mathrm{~A}}$ plt $A^{\mathrm{E} 133 \mathrm{~A}}$; Fig. 1). Otherwise, the wild-type and TN challenge strain variants displayed comparable phenotypic properties with regards to Vi-capsule expression, cellular invasion, in vitro growth characteristics, antibiotic susceptibility and survival in environmental water and soil samples (data not shown). Cell cycle arrest in vitro was observed with the wild-type but not the TN strain (Fig. 1).

We enrolled a total of 41 healthy adults (aged 18-60 years) into a randomized, double-blind, human challenge study between 10 April and 1 August 2017. One volunteer withdrew prior to challenge,

\footnotetext{
'Oxford Vaccine Group, Department of Paediatrics, University of Oxford and the NIHR Oxford Biomedical Research Centre, Oxford, UK. ${ }^{2}$ Department of Medicine, Imperial College London, London, UK. ${ }^{3}$ Nuffield Department of Primary Care Health Sciences, Clinical Trials Unit, University of Oxford, Oxford, UK. ${ }^{4}$ Wellcome Sanger Institute, Wellcome Genome Campus, Hinxton, UK. ${ }^{5}$ Department of Vector Biology, Liverpool School of Tropical Medicine, Liverpool, UK. ' ${ }^{6}$ uman Immune Monitoring Center, Institute for Immunity, Transplantation and Infection, Stanford University, Stanford, CA, USA. ${ }^{7}$ Department of Microbial Pathogenesis, Yale University School of Medicine, New Haven, CT, USA. ${ }^{8}$ Nuffield Department of Medicine, University of Oxford, Oxford, UK. ${ }^{\circ}$ Department of Medicine, University of Cambridge, Hinxton, UK. *e-mail: malick.gibani@paediatrics.ox.ac.uk
} 

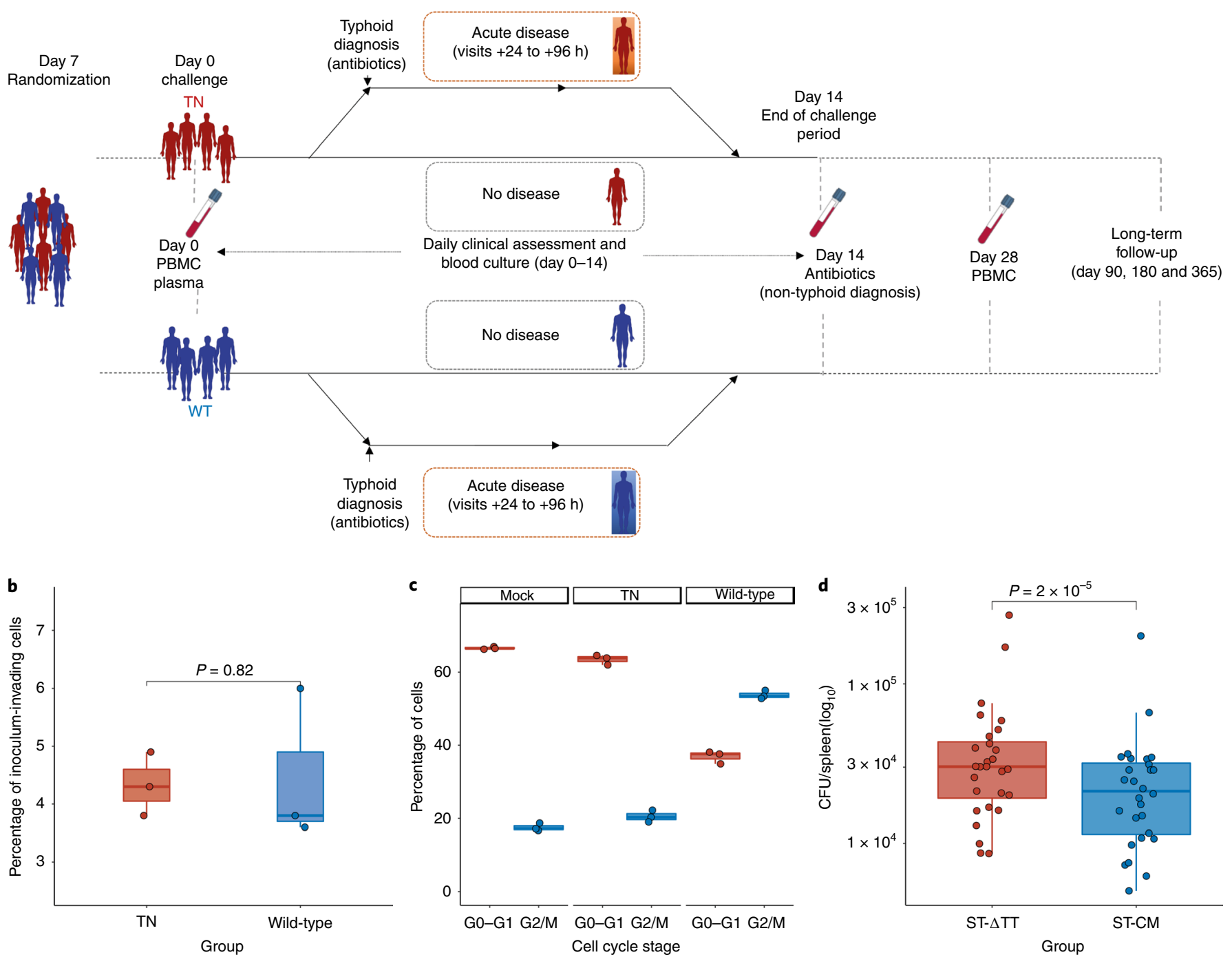

Fig. 1 | Trial design. a, Schematic of trial design (Supplementary Information); comparison of wild-type and TN strains. b, Cellular invasion assessed using a gentamicin protection assay. $\mathrm{MOI}=50, n=3$ independent replicates, two-sided Mann-Whitney U-test. c, Induction of cell cycle arrest in Henle-407 cells infected with the wild-type or TN strain $(\mathrm{MOI}=50)$. Intoxicated cells show a larger proportion of cells in the $\mathrm{G} 2 / \mathrm{M}$ phase of the cell cycle. $n=3$ independent replicates. d, Comparison of the bacterial loads of $S$. Typhi Quailes typhoid toxin-null mutant (ST- $\Delta \mathrm{TT}, \Delta \mathrm{pltB}, \Delta \mathrm{plt} A, \Delta \mathrm{cdtB}$ ) with $\mathrm{S}$. Typhi Quailes typhoid toxin catalytic mutant $\left(\mathrm{ST}-\mathrm{CM}, c d t B^{\mathrm{H} 160 \mathrm{Q}}, \mathrm{plt}^{\mathrm{S} 35 \mathrm{~A} A}, \mathrm{plt}^{\mathrm{E}}{ }^{\mathrm{E} 33 \mathrm{~A}}\right) . n=28$, Wilcoxon signed-rank test. The box plots display the median and IQR, with the upper whiskers extending to the largest value $\leq 1.5 \times \mathrm{IQR}$ from 75 th percentile and the lower whiskers extending to smallest values $\leq 1.5 \times \mathrm{IQR}$ from 25th percentile. The Fig. 1a images were sourced from Servier Medical Art and reproduced and adapted under a Creative Commons 3.0 unported license ${ }^{29}$.

and 40 completed the challenge protocol (Extended Data Fig. 1). The study was undertaken in a cohort of healthy adult volunteers in a setting non-endemic for typhoid fever (Oxford ${ }^{14}$; see the Life Sciences Reporting Summary). Groups were well matched at baseline (Extended Data Fig. 2 and Supplementary Data). Participants fasted for $90 \mathrm{~min}$ before oral challenge with $1-5 \times 10^{4}$ colony-forming units (CFUs) of either wild-type or TN strains administered $2 \mathrm{~min}$ after sodium bicarbonate pretreatment (Supplementary Information). Study visits were scheduled for $12 \mathrm{~h}$ after challenge, and then daily for $14 \mathrm{~d}$, when daily blood cultures were collected (Fig. 1) (ref. ${ }^{7}$ ). Antibiotic treatment (ciprofloxacin $500 \mathrm{mg}$ twice daily) was initiated at typhoid diagnosis or at day 14 for those without illness.

Using the primary composite diagnostic end point of fever $\geq 38^{\circ} \mathrm{C}$ for $\geq 12 \mathrm{~h}$ and/or $S$. Typhi bacteremia, we observed no significant difference in the rate of typhoid disease between participants challenged with wild-type or TN strains (15 out of 21 (71\%) versus 15 out of $19(79 \%)$; relative risk 1.11 (95\% confidence interval (CI) $0.8-1.6) ; P=0.58$; Fig. 2 and Supplementary Data). The attack rate $\left(n^{\text {diagnosed }} / n^{\text {challenged }}\right)$ in the wild-type group met the target range of $60-75 \%$ and was consistent with earlier studies ${ }^{7,13,14}$. There was no significant difference in the attack rate when we applied alternative diagnostic criteria (Supplementary Data). The challenge dose administered did not impact the outcome of the challenge (Fig. 2). Furthermore, there was no significant difference in time to diagnosis between wild-type and TN groups (median (interquartile range (IQR)) days to diagnosis $7.05(5.08-8.83)$ versus 5.25 (5.01-6.14); $P=0.23$; Fig. 2).

To determine if absence of the typhoid toxin was associated with an altered disease phenotype, we compared the clinical profiles between challenge groups (Fig. 2). Five participants met the prespecified criteria for severe typhoid fever; of these, one participant was randomized to wild-type ( 1 out of $15 ; 7 \%$ ) and four ( 4 out of $15 ; 27 \%$; $P=0.3$ ) were randomized to TN (Supplementary Data). Two serious 

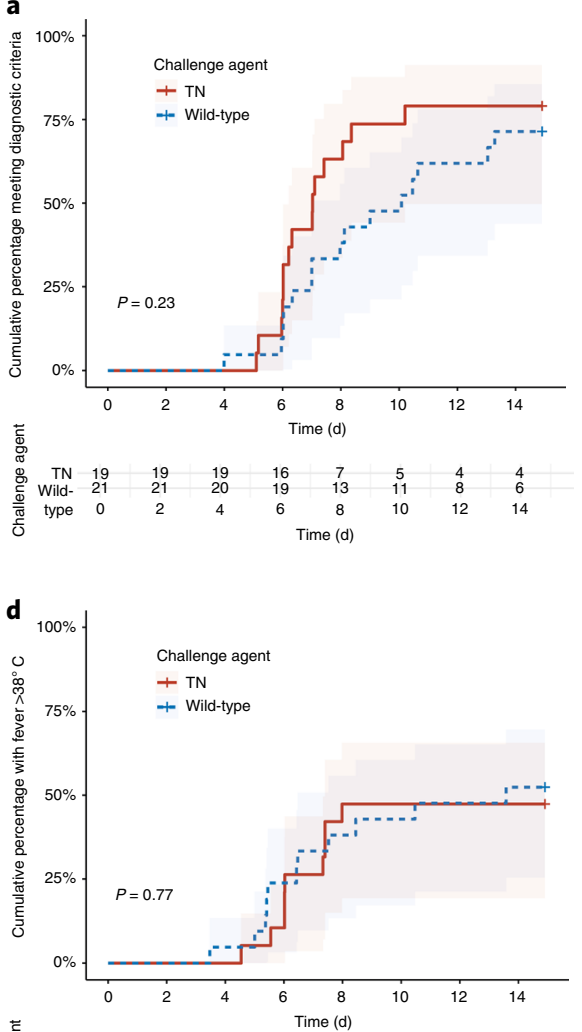

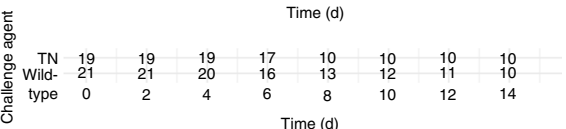

b

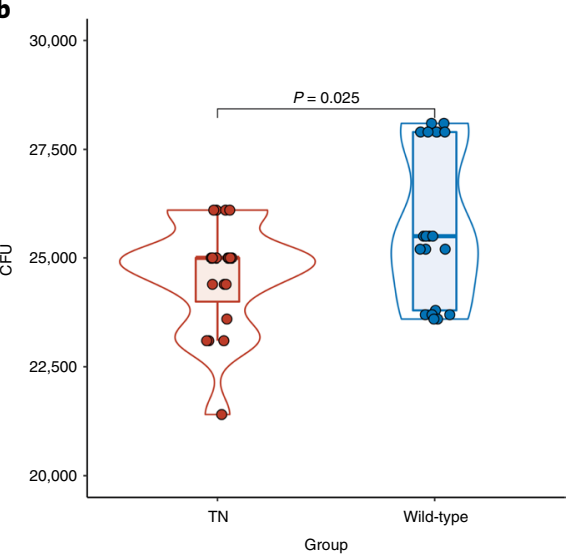

e

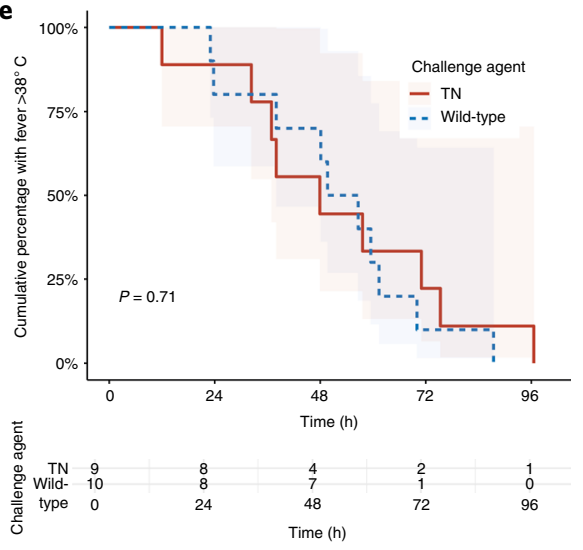

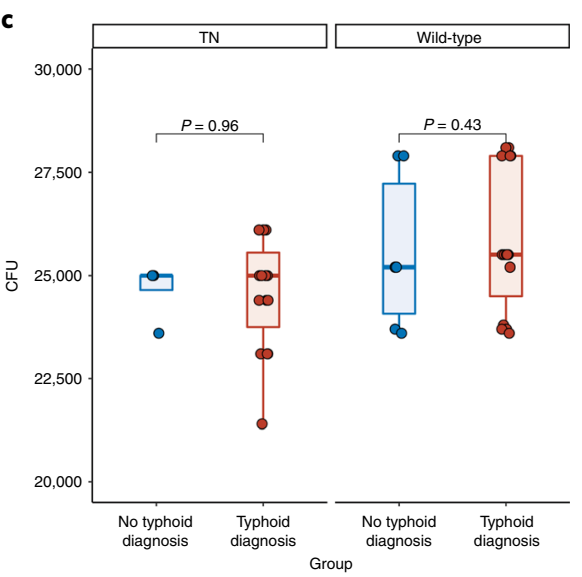

f

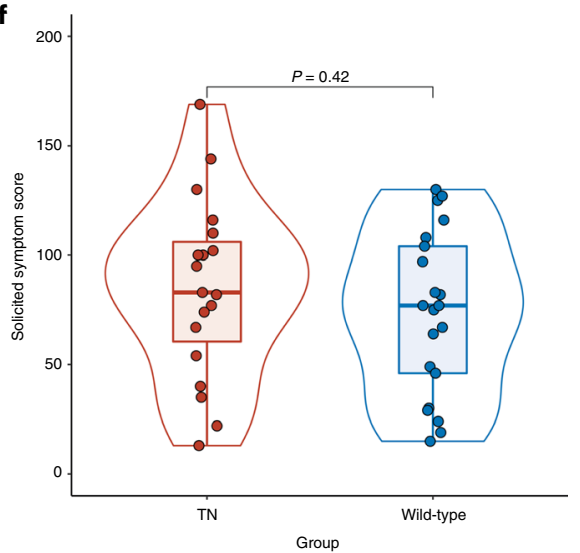

g

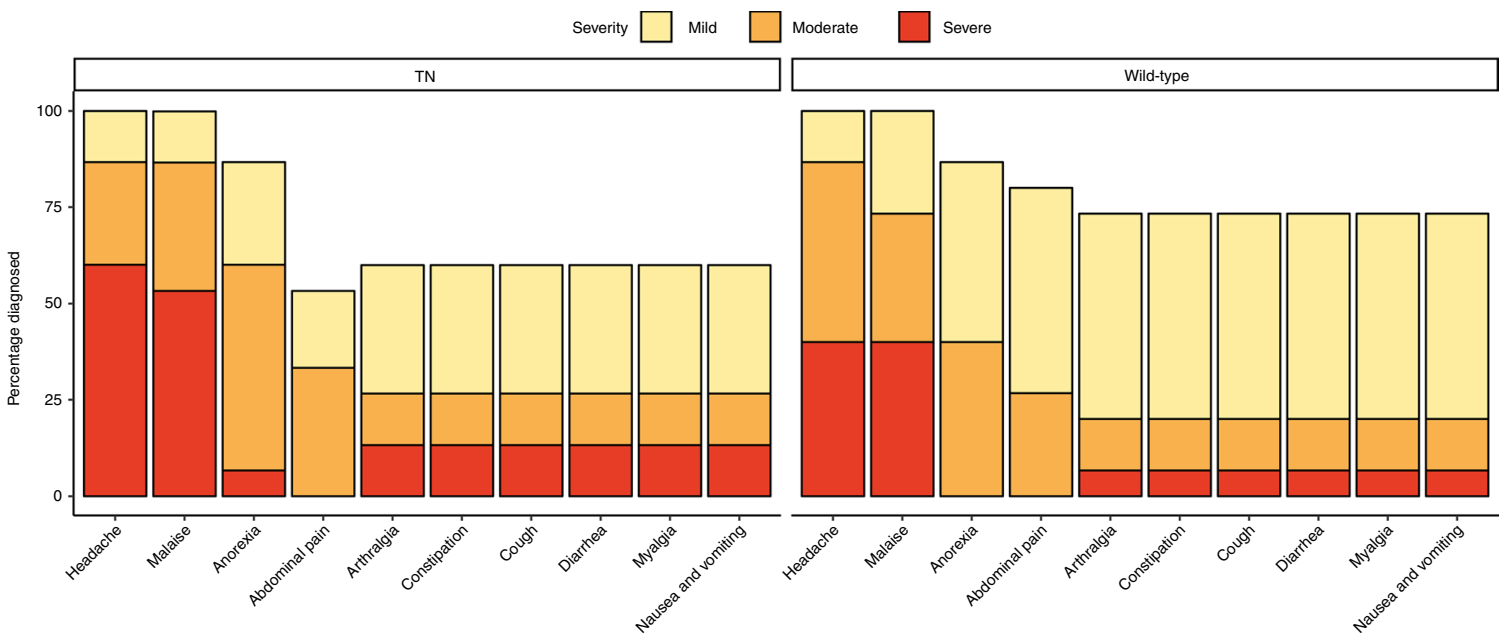

Solicited symptoms

Fig. 2 | Clinical response to challenge with wild-type and TN S. Typhi. a, Time to diagnosis after challenge. Cumulative proportion of participants meeting the composite diagnostic end point defined as $\mathrm{S}$. Typhi bacteremia and/or fever $\geq 38 \mathrm{C}^{\circ}$ persisting $\geq 12 \mathrm{~h}$. Participants not meeting the diagnostic criteria for typhoid diagnosis were censored at day 14 . log-rank test. b. Challenge dose administered between wild-type and TN challenge groups. Two-sided MannWhitney $U$-test, $n^{\text {TN }}=19, n^{\text {Wild-type }}=21$. c , Challenge dose administered according to outcome and challenge strain. Mann-Whitney U-test. d, Time to first fever $>38^{\circ} \mathrm{C}$. e, Fever clearance time categorized according to study group. Kaplan-Meier survival curve showing the cumulative proportion of participants with any fever $>38^{\circ} \mathrm{C}$ by challenge group. Participants with no recorded fever were censored at day 14 . log-rank test. f, Cumulative symptom severity scores in all participants challenged ${ }^{30} ; n^{\mathrm{TN}}=19, n^{\text {Wid-type }}=21$. Two-sided Mann-Whitney U-test. $\mathbf{g}$, Maximum symptom severity score (day 0-21) in participants diagnosed with typhoid fever according to study group. Percentage of participants reporting one or more events, graded as mild, moderate or severe ${ }^{30}$. The box plots display the median and IQR, with the upper whiskers extending to largest value $\leq 1.5 \times I Q R$ from the 75 th percentile and the lower whiskers extending to the smallest values $\leq 1.5 \times$ IQR from the 25 th percentile $^{31}$. The overlaid violin plots illustrate the distribution of the data points and their probability density ${ }^{31}$.

adverse events were reported, neither of which was assessed as being related to $S$. Typhi challenge (Supplementary Information). The most common symptoms reported by participants who developed typhoid were headache (30 out of 30;100\%), malaise (30 out of $30 ; 100 \%)$, anorexia ( 26 out of $30 ; 87 \%$ ) and abdominal pain (23 out of 30; 77\%; Fig. 2 and Supplementary Information). 
a

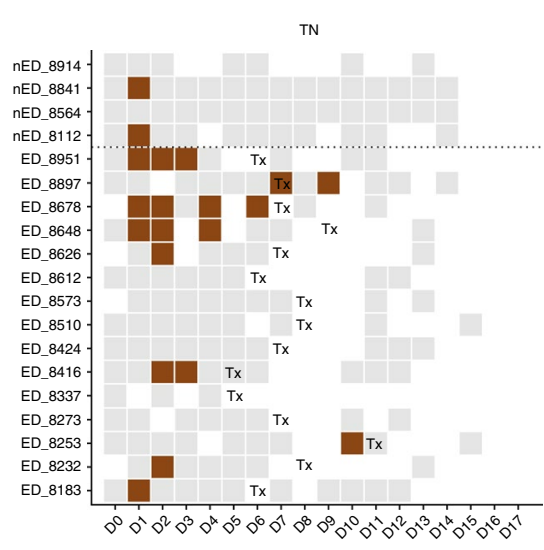

d

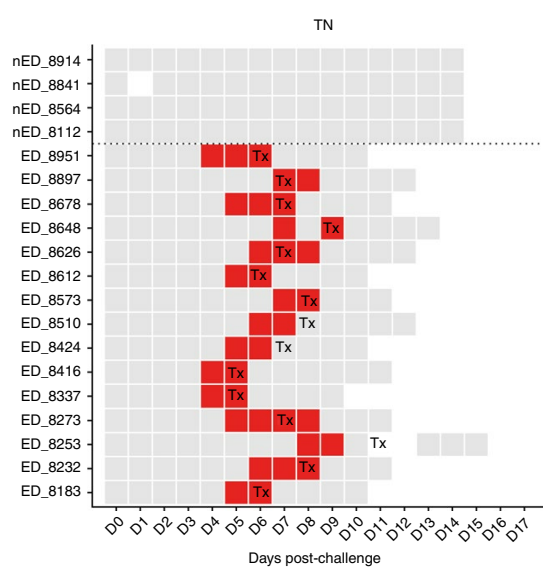

g

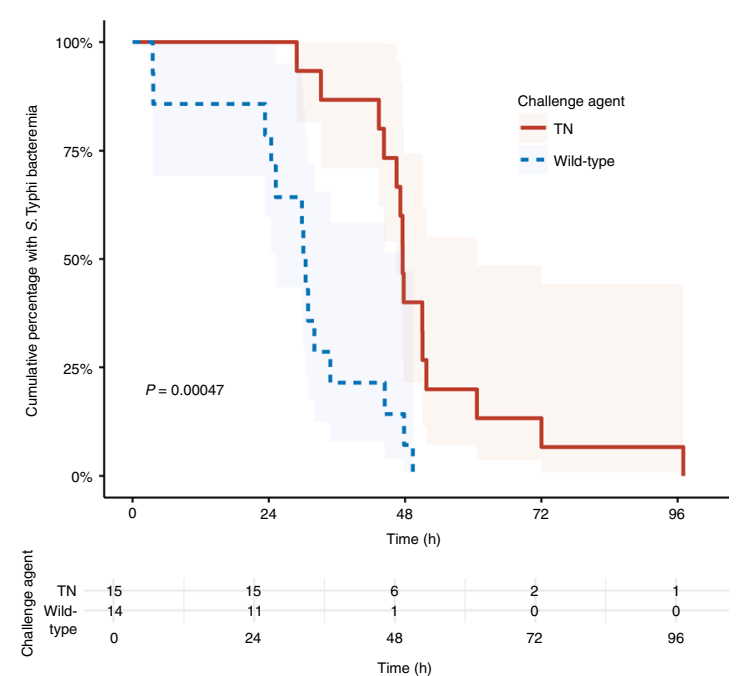

b

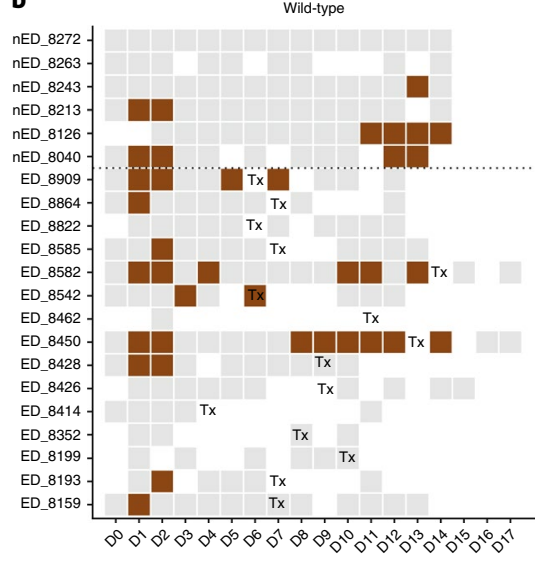

e

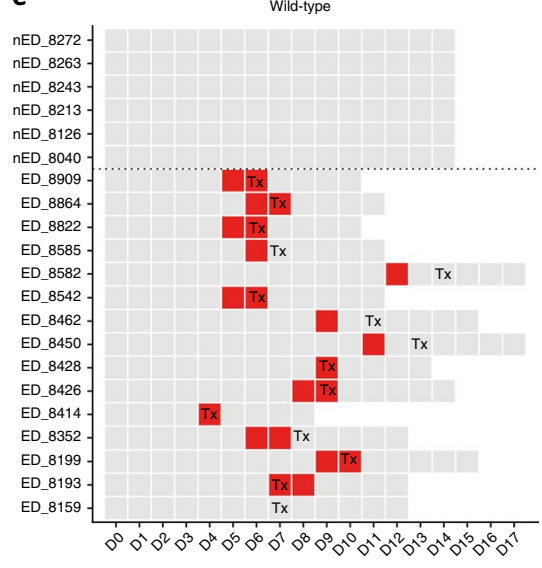

Days post-challenge c

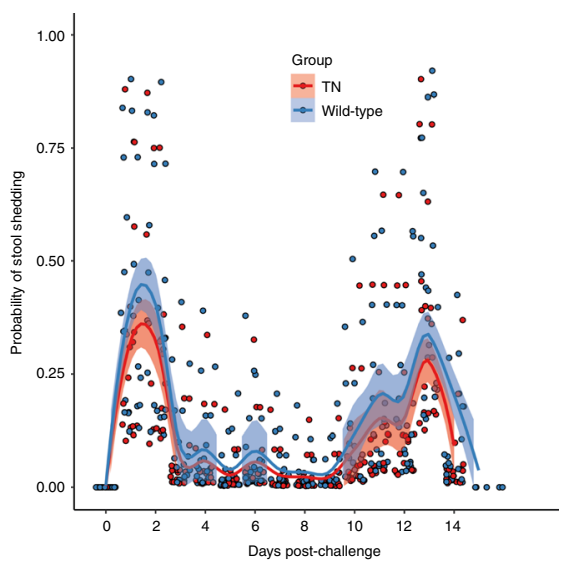

f

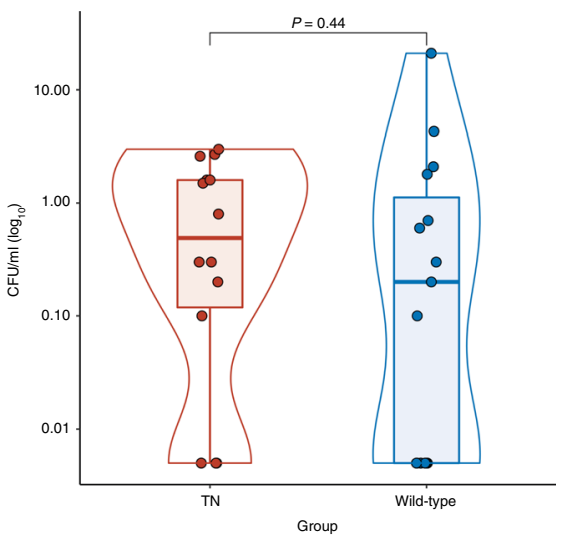

h

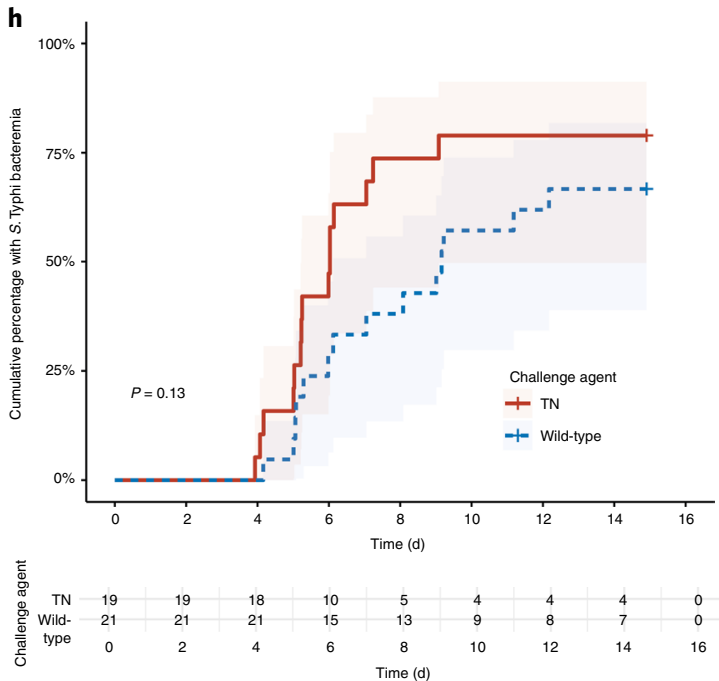

Fig. 3 | Microbiological response to challenge with wild-type and TN S. Typhi. a,b, Pattern of stool shedding after TN (a) and wild-type (b) challenge. The rows correspond to individual participants. Gray squares, negative sample; brown squares, positive stool culture; white squares = no sample collected. Tx is the day of treatment initiation. c, Probability of stool shedding S. Typhi over time after challenge. Samples were classified as culture-positive or culturenegative for S. Typhi and combined in mixed effects logistic regression models, as described previously ${ }^{18} . n^{\text {Wild-type }}=21, n^{\text {TN }}=19 . \mathbf{d}, \mathbf{e}$, Pattern of bacteremia after TN (d) and wild-type (e) challenge. Red squares= positive blood culture. Participants above the dotted lines did not meet the composite criteria for typhoid diagnosis. f, Quantitative blood culture at time of typhoid diagnosis. Samples with no colonies were assigned an arbitrary value corresponding to half the lower limit of detection $\left(0.05\right.$ CFU ml $\left.{ }^{-1}\right) . n^{\text {Wild-type }}=15, n^{\top N}=15$, two-sided Mann-Whitney $U$-test. g,h, Kaplan-Meier survival curve showing the cumulative proportion of participants with bacteremia after challenge ( $\mathbf{g}$ ) and time to S. Typhi bacteremia (h). Participants not meeting the diagnostic criteria were censored at day 14. Cumulative proportion of participants with ongoing bacteremia were measured from time of treatment initiation to first persistently negative blood culture, according to challenge group. log-rank test. The box plots display the median and IQR, the upper whiskers extending to the largest value $\leq 1.5 \times I Q R$ from the 75th percentile and the lower whiskers extending to the smallest values $\leq 1.5 \times I Q R$ from the 25 th percentile. The overlaid violin plots illustrate the distribution of the data points and their probability density ${ }^{31}$. 
a
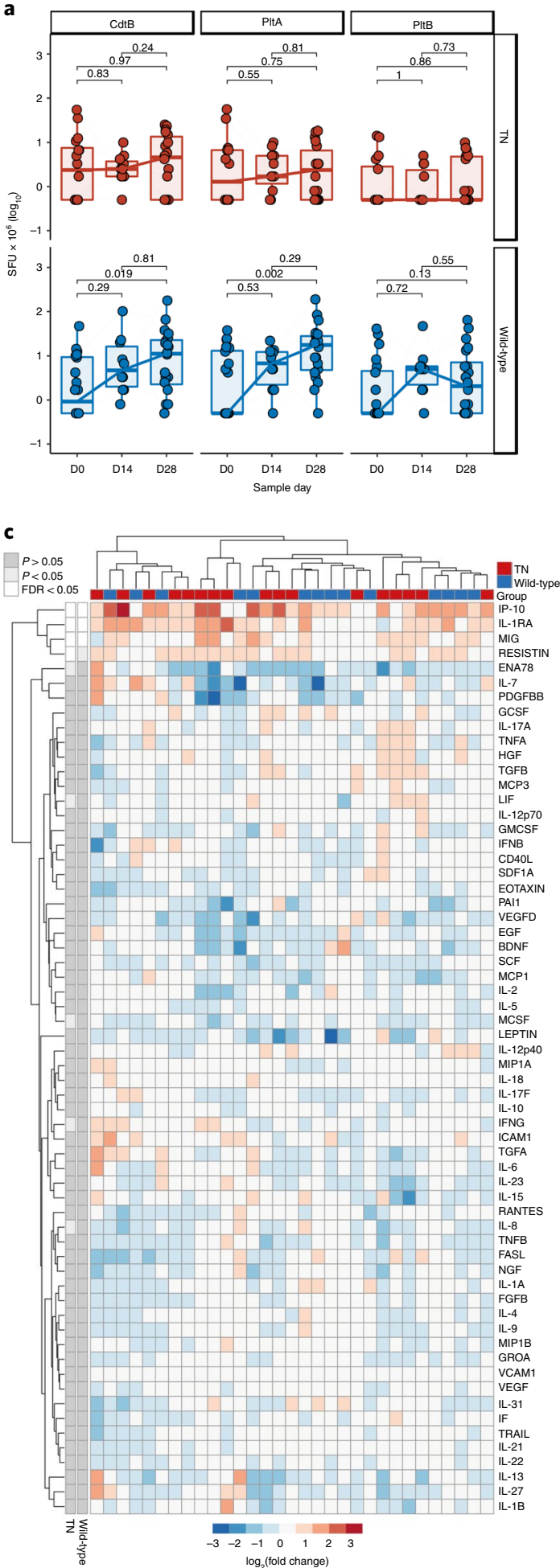
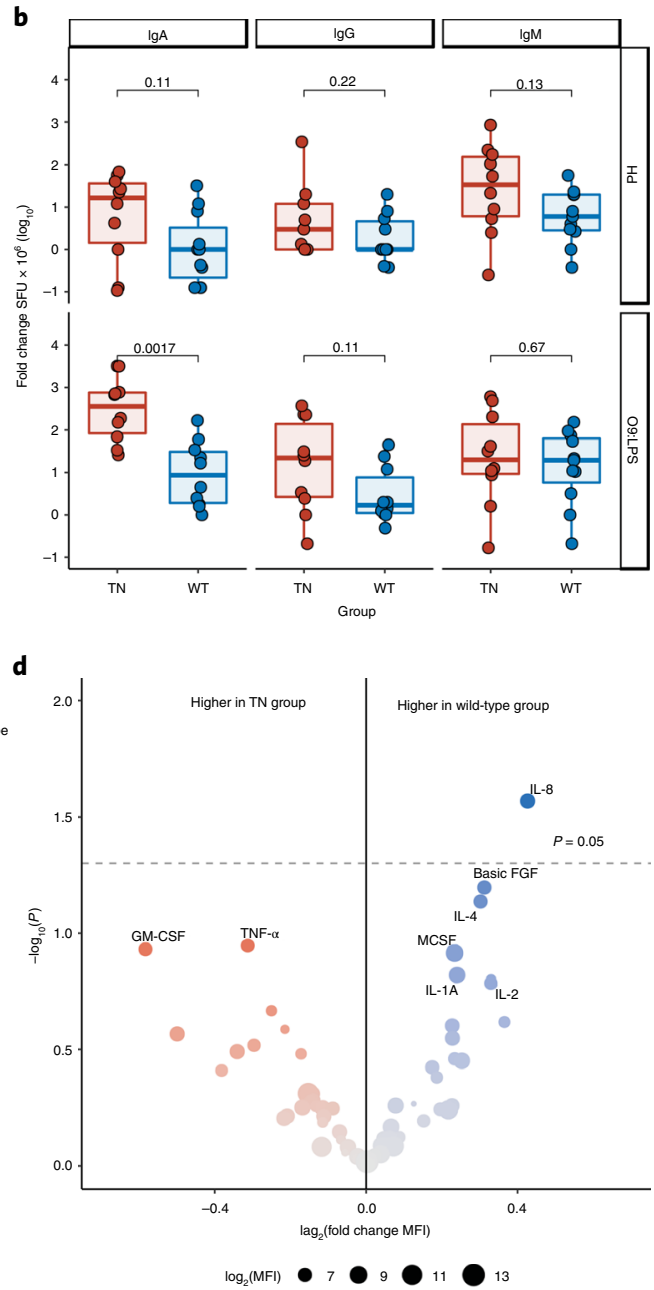

$\mathbf{e}$

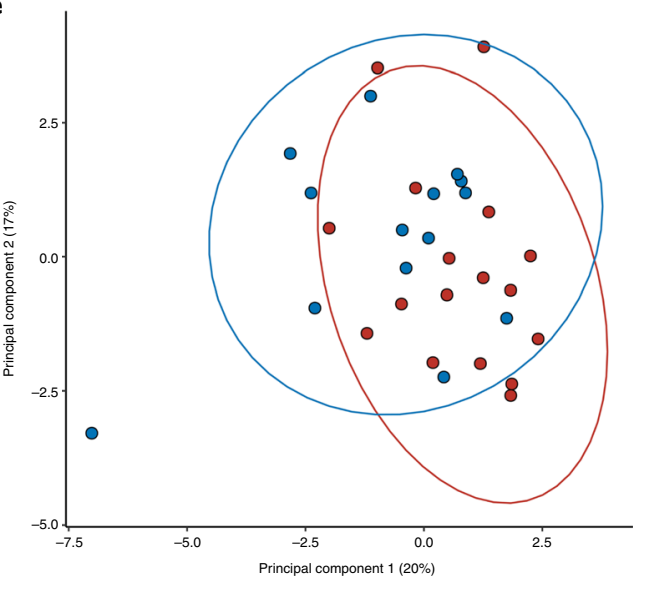

Fig. 4 | Host response to challenge with wild-type and TN S. Typhi. a, CdtB-, PItA- and PItB-specific IFN- $\gamma$-producing PBMCs at baseline, day 14 and 28 after challenge. $n^{\text {Wild-type }}=21, n^{\text {TN }}=19$, Wilcoxon signed-rank test for within-group comparisons on paired samples. $\mathbf{b}$, Magnitude of ASC response at typhoid diagnosis. $n^{\text {Wild-type }}=11, n^{\text {TN }}=10$; two-sided Mann-Whitney U-test. The box plots display the median and IQR. c, Plasma cytokine profiles after challenge with wild-type and TN S. Typhi. Heatmap showing $\log _{2}$ fold change in MFI for each cytokine (rows) and participant (columns) at time of diagnosis relative to baseline. $n^{\text {Wild-type }}=15, n^{\mathrm{TN}}=15$. Rows are annotated by significance of cytokine up- or downregulation relative to baseline in each challenge group (white, significant after adjustment for multiple testing; light gray, significant before adjustment; dark gray, non-significant). Two-sided moderated $t$-test with Benjamin-Hochberg correction. Clustering by Euclidean distance. FDR, false discovery rate. d, Volcano plots illustrate plasma cytokine up/downregulation at typhoid diagnosis in wild-type and TN challenge groups, with adjustment for baseline. The size of each point reflects average abundance $\left(\log _{2} \mathrm{MFI}\right)$ in the plasma. $n^{\text {Wild-type }}=15$, blue; $n^{\mathrm{TN}}=15$, red. e, Principal component plot of log fold change in MFI relative to baseline for each participant. Ellipses are drawn with a 95\% confidence level. $n^{\text {Wild-type }}=15, n^{\mathrm{TN}}=15$. FGFB, basic fibroblast growth factor; GM-CSF, granulocytemacrophage colony-stimulating factor; M-CSF, macrophage colony-stimulating factor. 
Fever clearance time was comparable between wild-type and TN groups (median (IQR) hours 53.15 (23.0-87.4) versus 44.92 (1296.6) hours; $P=0.71$; Fig. 2). Laboratory abnormalities (elevated C-reactive protein, lymphopenia, neutropenia) were all consistent with the expected presentation of typhoid fever in the field ${ }^{17}$ (Extended Data Fig. 3). Overall, the clinical phenotype was comparable between groups.

We next assessed if absence of typhoid toxin was associated with altered microbiological end points (Fig. 3). At least one stool culture was positive for $S$. Typhi in 13 out of 21 (62\%) participants challenged with the wild-type strain and 11 out of $19(58 \%)$ challenged with the TN strain. The pattern of stool shedding was comparable between groups, peaking $24-48 \mathrm{~h}$ after challenge, followed by a second peak in week 2 (Fig. 3) ( ref. $^{18}$ ). There was no difference in the probability of shedding over the entire challenge period following challenge with TN compared with wild-type (odds ratio $0.64,95 \%$ CI $0.17-2.47, P=0.51)$. In a humanized mouse model, infection with a typhoid toxin-deficient strain of $S$. Typhi was associated with an increased bacterial burden compared with the wild-type strain ${ }^{19}$. Consistent with this observation, the duration of bacteremia was significantly longer in participants challenged with the TN strain compared with the wild-type strain (47.6h (28.9-97.0) versus 30.3 (3.6-49.4); $P \leq 0.001$; Fig. 3), although circulating quantitative colony counts did not differ $\left(0.2 \mathrm{CFU} \mathrm{ml}^{-1}(0-21)\right.$ versus $0.55 \mathrm{CFU} \mathrm{ml}^{-1}$ $(0-3) ; P=0.44$; Fig. 3$)$. We next performed a principal component analysis of disease severity, using all clinical, microbiological and laboratory measures collected during the course of the challenge study. When all participants were included in the analysis, participants diagnosed with typhoid fever clearly cluster separately from individuals who did not develop disease (Extended Data Fig. 4); however, there was no clustering of participants by challenge group, suggesting that challenge with a typhoid toxin-deficient strain of $S$. Typhi was associated with an indistinguishable clinical phenotype to that caused by wild-type $S$. Typhi (Extended Data Fig. 5).

To determine if absence of the typhoid toxin modulated host immune responses to infection, we measured T-cell and antibody-secreting cell (ASC) responses between challenge groups. Interferon- $\gamma$ (IFN- $\gamma$ )-producing T-cell responses to peptide pools comprising the typhoid toxin subunits $\mathrm{CdtB}$, PltA and PltB were detectable in participants challenged with the wild-type strain, but not the TN strain, and peaked at day 28 post-challenge (Fig. 4). We observed a significant increase in circulating ASCs specific to the $S$. Typhi surface antigens O9:LPS and Hd at the time of typhoid diagnosis in both challenge groups (Extended Data Fig. 6) (ref. ${ }^{13}$ ). The magnitude of the O9:LPS-antigen- and Hd-antigen-specific ASC response at typhoid diagnosis was generally greater in participants challenged with the TN strain. In particular, S. Typhi O9:LPSspecific immunoglobulin A ASC responses at the time of typhoid diagnosis were significantly increased in the TN group (Fig. 4).

We next aimed to determine if the presence or absence of typhoid toxin was associated with a distinct plasma cytokine profile, measured using a 62-plex bead-based cytokine platform (Luminex) at baseline and during acute typhoid disease (Fig. 4). At the time of typhoid diagnosis, the plasma cytokines $10 \mathrm{kDa}$ interferon-gammainduced protein (IP-10), monokine induced by interferon-gamma (MIG) and interleukin-1 receptor antagonist protein (IL-1RA) were significantly increased relative to baseline in both groups (Fig. 4). Hierarchical clustering and principal component analysis showed no separation of challenge groups by cytokine profile during acute typhoid disease (Fig. 4). Following adjustment for multiple testing, linear modeling found no cytokines to be significantly different between groups, although interleukin 8 (IL-8) was marginally downregulated in the toxin-negative but not the wild-type group (Fig. 4).

These data suggest that the typhoid toxin is not essential for $S$. Typhi infection nor the early acute presentation of typhoid fever.
This study represents the first application of a typhoid human challenge model to prospectively study the role of a specific virulence factor in the pathogenesis of typhoid fever. Previous trials of liveattenuated $S$. Typhi vaccines have offered insights into the importance of other Salmonella genes to human disease (including aroC/ aroD, htrA, phoP, phoQ, ssaV and cya ${ }^{20}$. Overall, in this study, the clinical presentation was indistinguishable between the TN and wild-type groups. Counterintuitively, there was a trend toward a more severe disease phenotype in the TN group, including a shorter time to diagnosis, higher number of cases meeting the criteria for severe enteric fever, elevated ASC response and prolonged duration of bacteremia. These observations suggest that the typhoid toxin may have an important role in modifying host immune responses to infection.

These data raise questions as to the utility of targeting typhoid toxin in the development of novel therapeutics or vaccine strategies. Notably, currently utilized typhoid vaccines, including Vi capsular polysaccharide/conjugate vaccines, are capable of inducing protection despite targeting a virulence factor that is not strictly necessary for the establishment of enteric fever ${ }^{21-23}$. Antibody and T-cell responses to typhoid toxin components have been detected in patients with typhoid fever ${ }^{24-27}$. Further studies are required to correlate host responses to typhoid toxin with protection against disease and to further characterize its function in the context of natural $S$. Typhi infection.

We acknowledge the limitations of our experimental approach. Due to ethical considerations, this model is not suited to assess the role of typhoid toxin in severe typhoid fever, including typhoid encephalopathy, which has been associated with typhoid toxin in animal models ${ }^{4,8}$. The primary diagnostic criteria minimizes risk to study participants by early treatment initiation ${ }^{7}$, but could mask differences between groups by treating self-limiting disease. The study population may not be generalizable to typhoid endemic countries, owing to differences in prior immune-priming and/or baseline genetic differences ${ }^{28}$.

The absence of experiments showing reversion to virulence after complementation of the typhoid toxin genes in vitro is a limitation of this study. Additional studies with a strain expressing inactive components of typhoid toxin (for example, $c d t B^{\mathrm{H} 160 \mathrm{Q}}, p l t B^{\mathrm{S} 35 \mathrm{~A}}, p l t A^{\mathrm{E} 133 \mathrm{~A}}$ ) could address whether deletion of typhoid toxin genes is associated with an altered phenotype beyond loss of toxicity. Importantly, the study was underpowered to detect anything other than a large effect size $(80 \%$ power to detect at $72 \%$ relative reduction). Additional in vitro studies and deeper analysis of challenge samples are ongoing to further characterize the potential immunobiological role of typhoid toxin in the pathogenesis of typhoid fever.

These data indicate that typhoid toxin is not essential for the development of early acute typhoid fever within the context of a controlled human infection model. These data highlight some of the benefits and challenges of studying bacterial virulence factors using controlled human infection models, in particular for the screening of potential vaccine and therapeutic targets.

\section{Online content}

Any methods, additional references, Nature Research reporting summaries, source data, statements of code and data availability and associated accession codes are available at https://doi.org/10.1038/ s41591-019-0505-4.

Received: 3 February 2019; Accepted: 30 May 2019; Published online: 3 July 2019

\section{References}

1. Stanaway, J. D. et al. The global burden of typhoid and paratyphoid fevers: a systematic analysis for the Global Burden of Disease Study 2017. Lancet Infect. Dis. 19, 369-381 (2019). 
2. Galán, J. E. Typhoid toxin provides a window into typhoid fever and the biology of Salmonella Typhi. Proc. Natl Acad. Sci. USA 113 , 6338-6344 (2016).

3. Deng, L. et al. Host adaptation of a bacterial toxin from the human pathogen Salmonella Typhi. Cell 159, 1290-1299 (2014).

4. Song, J., Gao, X. \& Galán, J. E. Structure and function of the Salmonella Typhi chimaeric A(2)B(5) typhoid toxin. Nature 499, 350-354 (2013).

5. Del Bel Belluz, L. et al. The typhoid toxin promotes host survival and the establishment of a persistent asymptomatic infection. PLoS Pathog. 12, e1005528 (2016).

6. Haghjoo, E. \& Galán, J. E. Salmonella typhi encodes a functional cytolethal distending toxin that is delivered into host cells by a bacterial-internalization pathway. Proc. Natl Acad. Sci. USA 101, 4614-4619 (2004).

7. Waddington, C. S. et al. An outpatient, ambulant-design, controlled human infection model using escalating doses of Salmonella Typhi challenge delivered in sodium bicarbonate solution. Clin. Infect. Dis. 58, 1230-1240 (2014).

8. Yang, Y. A. et al. In vivo tropism of Salmonella Typhi toxin to cells expressing a multiantennal glycan receptor. Nat. Microbiol. 3, 155-163 (2018).

9. Parkhill, J. et al. Complete genome sequence of a multiple drug resistant Salmonella enterica serovar Typhi CT18. Nature 413, 848-852 (2001).

10. den Bakker, H. C. et al. Genome sequencing reveals diversification of virulence factor content and possible host adaptation in distinct subpopulations of Salmonella enterica. BMC Genomics 12, 425 (2011).

11. Rodriguez-Rivera, L. D., Bowen, B. M., den Bakker, H. C., Duhamel, G. E. \& Wiedmann, M. Characterization of the cytolethal distending toxin (typhoid toxin) in non-typhoidal Salmonella serovars. Gut Pathog. 7, 19 (2015).

12. Akinyemi, K. O. et al. Prevalence of multi-drug resistant Salmonella typhi among clinically diagnosed typhoid fever patients in Lagos, Nigeria. $Z$. Naturforsch. C 55, 489-493 (2000).

13. Darton, T. C. et al. Using a human challenge model of infection to measure vaccine efficacy: a randomised, controlled trial comparing the typhoid vaccines M01ZH09 with placebo and Ty21a. PLoS Negl. Trop. Dis. 10, e0004926 (2016).

14. Jin, C. et al. Efficacy and immunogenicity of a Vi-tetanus toxoid conjugate vaccine in the prevention of typhoid fever using a controlled human infection model of Salmonella Typhi: a randomised controlled, phase $2 \mathrm{~b}$ trial. Lancet 390, 2472-2480 (2017).

15. Wong, V. K. et al. An extended genotyping framework for Salmonella enterica serovar Typhi, the cause of human typhoid. Nat. Commun. 7, 12827 (2016).

16. Spanò, S., Ugalde, J. E. \& Galán, J. E. Delivery of a Salmonella Typhi exotoxin from a host intracellular compartment. Cell Host Microbe 3, 30-38 (2008)

17. Parry, C. M., Hien, T. T., Dougan, G., White, N. J. \& Farrar, J. J. Typhoid fever. N. Engl. J. Med. 347, 1770-1782 (2002).

18. Gibani, M. M. et al. The impact of vaccination and prior exposure on stool shedding of Salmonella Typhi and Salmonella Paratyphi in 6 controlled human infection studies. Clin. Infect. Dis. 68, 1265-1273 (2019).

19. Song, J. et al. A mouse model for the human pathogen Salmonella Typhi. Cell Host Microbe 8, 369-376 (2010).

20. Tennant, S. M. \& Levine, M. M. Live attenuated vaccines for invasive Salmonella infections. Vaccine 33, C36-C41 (2015)

21. Milligan, R., Paul, M., Richardson, M. \& Neuberger, A. Vaccines for preventing typhoid fever. Cochrane Database Syst. Rev. 5, CD001261 (2018).

22. Baker, S. et al. Detection of Vi-negative Salmonella enterica serovar typhi in the peripheral blood of patients with typhoid fever in the Faisalabad region of Pakistan. J. Clin. Microbiol. 43, 4418-4425 (2005)

23. Maskey, A. P. et al. Salmonella enterica serovar Paratyphi A and S. enterica serovar Typhi cause indistinguishable clinical syndromes in Kathmandu, Nepal. Clin. Infect. Dis. 42, 1247-1253 (2006)

24. Liang, L. et al. Immune profiling with a Salmonella Typhi antigen microarray identifies new diagnostic biomarkers of human typhoid. Sci. Rep. 3, 1043 (2013).

25. Chin, C. F. et al. Delineation of B-cell epitopes of Salmonella enterica serovar Typhi hemolysin E: potential antibody therapeutic target. Sci. Rep. 7, 2176 (2017).

26. Sharma, T. et al. Serodiagnostic evaluation of recombinant CdtB of $S$. Typhi as a potential candidate for acute typhoid. Immunol. Res. 66, 503-512 (2018).

27. Darton, T. C. et al. Identification of novel serodiagnostic signatures of typhoid fever using a Salmonella proteome array. Front. Microbiol. 8, 1794 (2017).

28. Dunstan, S. J. et al. Variation at HLA-DRB1 is associated with resistance to enteric fever. Nat. Genet. 46, 1333-1336 (2014).

29. Creative Commons Attribution 3.0 Unported; https://creativecommons.org/ licenses/by/3.0/legalcode.
30. Dobinson, H. C. et al. Evaluation of the clinical and microbiological response to Salmonella paratyphi A infection in the first paratyphoid human challenge model. Clin. Infect. Dis. 64, 1066-1073 (2017).

31. Wickham, H., Chang, W. \& Henry, L. Package 'ggplot2'. (2019). R package version 3.2.0 https://cran.r-project.org/web/packages/ggplot2/ggplot2.pdf (2019)

\section{Acknowledgements}

The authors wish to acknowledge the contribution of all study participants. The authors acknowledge the support of the Wellcome Trust (Wellcome Trust Strategic Translational Award grant no. 092661) in the development of the typhoid human challenge model that was used for this study, and the support of the NIHR Oxford Biomedical Research Centre. Cytokine studies were supported by the Bill \& Melinda Gates Foundation: Global Health Vaccine Accelerator Platform grant to the Center for Human Systems Immunology at Stanford (no. OPP1113682). M.M.G. is supported in part by the NIHR Imperial Biomedical Research Centre. In addition, the authors wish to thank the following persons: R. Milward and the Walter Reed Army Institute of Research Pilot BioProduction Facility, who manufactured the GMP challenge lots; the Data Safety Monitoring Committee (D. Lalloo, D. Hill, P. Monk); M. Morgan and the microbiology laboratory at the Oxford University Hospital NHS foundation Trust; M. McClure; M. Raymond; T. Darton; C. Waddington; M. M. Levine; and the University of Maryland for provision of the original S. Typhi Quailes challenge strain. The study was funded by the Bill \& Melinda Gates Foundation (no. OPP1126235). The study funders had no role in study design, data collection or analysis.

\section{Author contributions}

J.G. and A.J.P. conceived the project. M.M.G. and A.J.P. designed the clinical study. M.M.G., C.Jin., J.M., D.C., E.J., S.C., C.J.B., C.Dold, C.Darlow, L.B., J.H., H.T.-B. and L.S.R. collected the data. M.M.G, J.M, C.Black and C.Jones co-ordinated study approvals and recruitment. The plasma cytokine assays were performed by Y.H.-R. and G.O. A.B. performed the cytokine analysis. M.L.-T. and X. J. constructed the typhoid toxin mutant strain and carried out its in vitro characterization. G. S. conducted the mouse infection studies. E.H. and G.D. analyzed the sequencing data. U.G. provided the statistical oversight. B.A. and. A.J.P provided clinical oversight. M.M.G. wrote the first draft of the manuscript and all authors reviewed and edited the manuscript and approved the final version.

\section{Competing interests}

A.J.P. chairs the UK Department of Health's (DH) Joint Committee on Vaccination and Immunisation (JCVI) and the European Medicines Agency Scientific Advisory Group on Vaccines, and is a member of the World Health Organization's (WHO) Strategic Advisory Group of Experts. A.J.P. previously received grant funding from Okairos, which ended in 2016. The views expressed in this manuscript are those of the authors and do not necessarily reflect the views of the JCVI, DH or WHO. All other authors declare no competing interests.

\section{Additional information}

Extended data is available for this paper at https://doi.org/10.1038/s41591-019-0505-4. Supplementary information is available for this paper at https://doi.org/10.1038/ s41591-019-0505-4.

Reprints and permissions information is available at www.nature.com/reprints. Correspondence and requests for materials should be addressed to M.M.G. Peer review information: Alison Farrell is the primary editor on this article and managed its editorial process and peer review in collaboration with the rest of the editorial team.

Publisher's note: Springer Nature remains neutral with regard to jurisdictional claims in published maps and institutional affiliations.

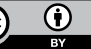

Open Access This article is licensed under a Creative Commons Attribution 4.0 International License, which permits use, sharing, adaptation, distribution and reproduction in any medium or format, as long as you give appropriate credit to the original author(s) and the source, provide a link to the Creative Commons license, and indicate if changes were made. The images or other third party material in this article are included in the article's Creative Commons license, unless indicated otherwise in a credit line to the material. If material is not included in the article's Creative Commons license and your intended use is not permitted by statutory regulation or exceeds the permitted use, you will need to obtain permission directly from the copyright holder. To view a copy of this license, visit http://creativecommons.org/licenses/by/4.0/.

(C) The Author(s), under exclusive licence to Springer Nature America, Inc. 2019 


\section{Methods}

Study design and participants. The OVG2016/03 (TYGER) study was a randomized, double-blind, controlled human infection study comparing the response to challenge with wild-type $S$. Typhi with a typhoid toxin-deficient isogenic mutant strain $S$. Typhi (SB6000). The study was designed as an outpatient challenge study, conducted in a cohort of healthy community adult volunteers in a setting non-endemic for typhoid fever (Oxford).

Challenge strains. To facilitate comparisons with earlier challenge studies ${ }^{7,13,14}$ and to minimize the risk to study participants, we used the $S$. Typhi Quailes strain (genotype 3.0.1 (ref. ${ }^{15}$ )) as the parent strain to generate the typhoid toxin-deficient knockout strain. Deletion of typhoid toxin subunit genes was carried as described previously ${ }^{16}$

Briefly, deletion of typhoid toxin subunit genes was carried out using the R6Kderived, suicide vector pSB890. The pSB890 plasmid cannot replicate in S. Typhi since it requires the bacteriophage $\lambda$ pir protein to replicate. The plasmid vector also encodes a counterselectable marker $s a c B$, which encodes an enzyme that is lethal to bacteria when grown in the presence of sucrose. The pSB890 plasmid vector is maintained in a specially constructed strain of Escherichia coli, which encodes the bacteriophage $\lambda$ pir protein. This $E$. coli strain also carries a deletion mutation in the asd gene $(\Delta a s d)$, which encodes the aspartate-semialdehyde dehydrogenase required for peptidoglycan synthesis-growth of this strain will only occur in media supplemented with lysine diaminoheptanedioate.

Due to the genomic organization of the typhoid toxin pathogenicity island, the $c d t B$ gene was deleted first, followed by simultaneous deletion of the $p l t A$ and $p l t B$ genes (encoded immediately adjacent to one another) in the $\Delta c d t B$ strain. Chromosomal DNA fragments encoding sequences upstream and downstream of the target genes were expanded by PCR and cloned into the pSB890 plasmid, maintained in the E. coli $\Delta a s d \lambda$ pir strain. The plasmid vector encoding the cloned sequences was then transferred to $S$. Typhi by conjugation, counterselecting the donor E. coli strain by plating the transconjugants in media lacking lysine diaminoheptanedioate (counterselecting for the donor E. coli). Transconjugants of $S$. Typhi possessing deletions of the toxin integrated into the chromosome were identified by plating in sucrose that counterselects for the plasmid vector. Colonies were screened by PCR to identified mutants carrying the specific deletions.

Challenge strains were manufactured to a GMP standard at the Walter Reed Army Institute of Research (Silver Spring) and stored as a frozen suspension in soya tryptone medium containing $10 \%$ sucrose at $-80^{\circ} \mathrm{C}$ before use.

Strain characterization. Growth curves of wild-type and toxin-deficient strains of $S$. Typhi were performed in lysogeny broth (LB) using wild-type and TN strains. Isolates were inoculated into $10 \mathrm{ml}$ of LB and grown overnight in a shaking incubator at $200-220$ r.p.m. and $37^{\circ} \mathrm{C}$. The following day, cultures were vortexed and diluted 1:10 in fresh LB $(100 \mu \mathrm{l}$ culture $+900 \mu \mathrm{LB})$. The OD600 was read in a cuvette using a mini photospectrometer against an LB-only blank, multiplying by the dilution factor $(\times 10)$ to give the actual OD of the overnight culture. The dilution required to reduce the culture OD to 0.05 in a $30 \mathrm{ml}$ volume of $\mathrm{LB}$ was calculated using the following equation (equation (1)):

\section{$\mathrm{OD} 1 \times \mathrm{V} 1=\mathrm{OD} 2 \times \mathrm{V} 2$}

where OD1 is the OD of the overnight culture, V1 is the volume of the overnight culture to be added to the new mix; OD2 is theOD of the new inoculum $(0.05)$ and $\mathrm{V} 2$ is the volume of new inocula $(30 \mathrm{ml})$. The calculated volume of overnight cultures (V1) was added to $30 \mathrm{ml}$ of fresh LB, the OD600 read to ensure the OD of the new culture was 0.05 and was subsequently returned to the shaking incubator. Samples of culture were removed from the incubator at regular intervals and the OD600 was measured in a 50:50 mix of the culture and fresh LB against an LB blank. OD600 readings were multiplied by the dilution factor $(\times 2)$ to give values for the undiluted culture and plotted against time to give the growth curve.

Typhoid toxin activity. Activity of the typhoid toxin was assessed using previously published methods ${ }^{4,6}$. Briefly, Henle-407 intestinal epithelial cells were infected with wild-type Quailes or typhoid toxin-deficient SB6000 S. Typhi for $1 \mathrm{~h}$. Cells were washed and culture medium containing gentamicin $\left(50 \mu \mathrm{g} \mathrm{ml}^{-1}\right)$ was added and then incubated for $2 \mathrm{~h}$. Cell were then washed and medium containing $5 \mu \mathrm{g} \mathrm{ml}^{-1}$ gentamicin was added and infection continued for $48 \mathrm{~h}$. Cells were collected from dishes by trypsinization (subsequently neutralized with serumcontaining media). The cell suspensions were centrifuged for $5 \mathrm{~min}$ at 1500 r.p.m. the supernatant discarded and cell pellets resuspended in $0.5 \mathrm{ml}$ of PBS at room temperature. Cell suspensions were slowly added to tubes containing $4 \mathrm{ml}$ of cold $90 \%$ ethanol solution with continuous mixing. Cells were kept in fixative for $2 \mathrm{~h}$ on ice. The fixed cells were collected by centrifugation and the fixative decanted thoroughly. The pellets were washed once with $5 \mathrm{ml}$ PBS and the cell pellet was resuspended in $1 \mathrm{ml}$ of a solution containing $0.1 \%$ Triton X-100, DNase-free ribonuclease A $\left(20 \mathrm{mg} \mathrm{ml}^{-1}\right)$ and propidium iodide $\left(20 \mu \mathrm{g} \mathrm{ml}^{-1}\right.$; Molecular Probes) in PBS. The stained cells were analyzed by flow cytometry with a FACStar Plus flow cytometer (BD Biosciences). Intoxicated cells showed a larger proportion of cells in the G2/M phase of the cell cycle and thus exhibited a larger amount of DNA content.
Cellular invasion assay. Henle-407 intestinal epithelial cells were infected with wild-type S. Typhi Quailes or the toxin-deficient SB6000 derivative for $2 \mathrm{~h}$ at three multiplicity of infection (MOI) levels (50,100 and 500). Cells were washed and gentamicin $\left(50 \mu \mathrm{g} \mathrm{ml}^{-1}\right)$ was added to the culture medium. After $2 \mathrm{~h}$, cells were washed again, lysed and colony counts of both strains were determined by plating dilutions of the cell lysates. The invasive ability was expressed as the percentage of the bacterial inoculum that survived gentamicin treatment.

Comparison of the bacterial loads of $S$. Typhi Quailes $\Delta \mathrm{pltB}, \Delta$ pltA and $\Delta \mathrm{cdtB}$ with $S$. Typhi Quailes $c d t B^{\mathrm{H} 160 \mathrm{Q}}$, plt $B^{\mathrm{S} 35 \mathrm{~A}}$ and $p l t A^{\mathrm{E} 133 \mathrm{~A}}$. Strains used for comparison of the bacterial loads of $S$. Typhi Quailes $\Delta$ pltB, $\Delta$ pltA and $\Delta c d t B$ with $S$. Typhi Quailes $c d t B^{\mathrm{H} 160 \mathrm{Q}}$, plt $B^{\mathrm{S} 35 \mathrm{~A}}$ and $p l t A^{\mathrm{E} 133 \mathrm{~A}}$ were derived from $S$. Typhi Quailes and were constructed by standard recombinant DNA techniques as described previously ${ }^{16} . \mathrm{CmaH}^{-/-}$bloc $3^{-/-}$mice, which are susceptible to $S$. Typhi infection ${ }^{32}$, were intraperitoneally infected with equal numbers $\left(10^{5} \mathrm{CFUs}\right)$ of $S$. Typhi Quailes derivative mutant strains carrying either deletions in the pltB, plt $A$ and $c d t B$ genes (S. Typhi Quailes $\Delta p l t B, \Delta p l t A$ and $\Delta c d t B$ ) or expressing an inactivated version of typhoid toxin by virtue of catalytic mutations in its active subunits PltA and CdtB and a mutation in the receptor-binding site of PltB ( $S$. Typhi Quailes $c d t B^{\mathrm{H} 160 \mathrm{Q}}$, $p l t B^{\mathrm{S} 35 \mathrm{~A}}$ and $\left.p l t A^{\mathrm{E} 133 \mathrm{~A}}\right)$. The strains were alternatively marked by a chloramphenicol $(\mathrm{cmR})$ or kanamycin $(\mathrm{kanR})$ resistance genes, as indicated, inserted within the STY4607 gene, which previous studies have shown not to affect virulence ${ }^{32}$. All animal experiments were conducted in accordance with protocols approved by Yale University's Institutional Animal Care and Use Committee. Seven-to-ten week old $\mathrm{cmaH}^{-1-}$ bloc $3^{-/-}$mice were injected intraperitoneally with $10^{5} \mathrm{CFUs}$ each of the two strains. The inoculum was plated to confirm the equivalent ratio of the bacterial strains. Mice were killed at day 5 post-infection and the CFUs of each strain in the spleens of infected animals were determined by plating on LB plates containing chloramphenicol $\left(30 \mu \mathrm{g} \mathrm{ml}^{-1}\right)$ or kanamycin $\left(50 \mu \mathrm{g} \mathrm{ml}^{-1}\right)$.

Phenotypic characterization of the wild-type and TN strains comprised growth characteristics in liquid culture, agglutination, cellular invasion assays and cell intoxication assays ${ }^{4}$. Whole-genome sequencing using both the MiSeq (Illumina) and PacBio (Pacific Biosciences) platforms was performed by the Wellcome Sanger Institute (Hinxton). DNA for MiSeq sequencing was extracted using the Wizard Genomic DNA purification kit according to the manufacturer's instructions ${ }^{33}$. Sequence reads were assembled using HGAP v.3 of the SMRT analysis software v.2.3.0 (Supplementary Information).

Establishment of challenge dose. Challenge agents were prepared in batches for a maximum of six participants at any one time. All work was performed in the containment level 3 facility at the Centre for Clinical Vaccinology and Tropical Medicine (Oxford) in a class II biological safety hood dedicated for challenge agent preparation.

Two GMP master stock vials of typhoid toxin-negative $S$. Typhi TN strain (BPR-1218-00, lot 1977; cell concentration $1 \times 10^{6}$ ) or wild-type $S$. Typhi Quailes strain (BPR-1218-00, lot 1977; cell concentration $9.8 \times 10^{5}$ ) were selected at random from stocks stored in a $-80^{\circ} \mathrm{C}$ freezer. Vials were thawed at room temperature for approximately $10 \mathrm{~min}$ and mixed by vortexing. The contents of two GMP master stock vials were transferred to a master stock tube and mixed for 6-10s by vortexing. A 1:10 dilution in sodium bicarbonate was performed by transferring $1,600 \mu \mathrm{l}$ from the tube labeled 'master stock' to a fresh $50 \mathrm{ml}$ falcon labeled as 'master stock 1:10'. To create the challenge inoculum of the toxinnegative strain, $1.74 \mathrm{ml}$ from the 'master stock 1:10 dilution' was transferred to a sterile culture flask containing $4.2 \mathrm{~g}$ sodium bicarbonate dissolved in $240 \mathrm{ml}$ bottled mineral water ('challenge flask'). The challenge inoculum for the wild-type strain was generated by transferring $1.85 \mathrm{ml}$ from the 1:10 dilution into an equivalent challenge flask. The challenge agents were then prepared by transferring $30 \mathrm{ml}$ from the challenge flask to prelabelled $50 \mathrm{ml}$ falcon tubes, sealed and stored on ice.

The challenge dose was confirmed by pipetting $200 \mu \mathrm{l}$ from the challenge dose onto six Tryptone Soya Agar plates (code no. PO0163A; Oxoid). The bacterial suspension was spread over the source of the agar using an L-shaped spreader and cultured in an incubator overnight at $37^{\circ} \mathrm{C}, 5 \% \mathrm{CO}_{2}$. On the following day, colonies were manually counted and checked by a second operator. The CFUs of the challenge inoculum were calculated by multiplying the mean of the CFU counts for the plates by the dilution factor of the volume plated $(\times 150$ for a total challenge inoculum of $30 \mathrm{ml}$ for a plating of $0.2 \mathrm{ml}(30 / 0.2=\times 150)$ ).

Sodium bicarbonate was prepared by dissolving $2.1 \mathrm{~g}$ sodium bicarbonate in $120 \mathrm{ml}$ bottled mineral water.

Participant characteristics. Healthy adults aged $18-60$ years, without prior residency in an enteric fever endemic country for $\geq 6$ months, were considered eligible for enrollment. Key exclusion criteria included significant medical, surgical or psychiatric history and gallbladder disease. A full description of the inclusion and exclusion criteria is provided in the Nature Research Reporting Summary.

Randomization and masking. Participants were randomized 1:1 to challenge with either wild-type strain $S$. Typhi or toxin-negative strain $S$. Typhi (TN) in varying block sizes. Anti-Vi IgG was measured at screening using a commercial ELISA kit (VaccZyme; The Binding Site Ltd) according to the manufacturer's instructions ${ }^{14}$. 
Randomization was stratified by anti-Vi IgG measured (low $\left(<7.4 \mathrm{EU} \mathrm{ml}^{-1}\right)$ or high $\left.\left(\geq 7.4 \mathrm{EU} \mathrm{ml}^{-1}\right)\right)$. The exception was a sentinel group of two participants who were randomized 1:1 to receive the wild-type strain or TN knockout strain using a block size of two. Randomization was performed at the prechallenge visit, one week before challenge. We generated a randomization list in STATA v.14.2 (StataCorp), which was implemented in the computerized randomization software Sortition (Nuffield Department of Primary Care, Clinical Trials Unit, University of Oxford), which matched a masked allocation group to each participant. The software generated a randomization number, corresponding to the challenge allocation group. A locked, challenge agent randomization allocation list was maintained by the study statistician and unblinded laboratory team responsible for challenge agent preparation.

The study was conducted double-blind from the time of randomization until participant unblinding, such that participants, and clinical or laboratory staff undertaking follow-up procedures, were unaware of challenge agent allocation. Both wild-type and TN strains were prepared suspended in sodium bicarbonate and had an indistinguishable appearance (transparent, colorless liquid)

Procedures. Participants fasted for $90 \mathrm{~min}$ before challenge. Two minutes before

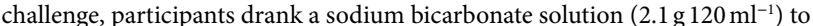
neutralize stomach acid. The oral challenge inoculum was administered suspended

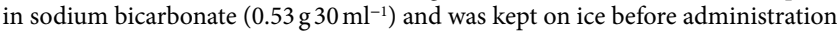
within $3 \mathrm{~h}$ of preparation. Participants were observed for $90 \mathrm{~min}$ post-challenge. The challenge dose administered was $1-5 \times 10^{4}$ CFUs calculated as described previously $y^{7,13,14}$. Participants attended the clinical site $12 \mathrm{~h}$ after challenge and then daily for $14 \mathrm{~d}$, as described previously ${ }^{7}$. Daily visits comprised continued consent check, oral temperature measurement, heart rate and blood pressure measurement and sample collection, as outlined in the study protocol

Solicited symptoms and twice-daily temperature measurements were recorded in an electronic diary for $21 \mathrm{~d}$ after challenge. Symptoms were categorized as not present, mild, moderate or severe (Supplementary Information).

Antibiotic treatment was initiated on fulfillment of composite diagnostic criteria or at day 14 for those without illness. First-line treatment was oral ciprofloxacin $500 \mathrm{mg}$ twice daily for $14 \mathrm{~d}$.

Outcomes. The primary objective of this study was to compare the proportion of participants meeting the composite diagnostic end point for typhoid fever (attack rate) following oral challenge with $(1-5) \times 10^{4}$ CFUs wild-type $S$. Typhi Quailes strain, compared to challenge with $(1-5) \times 10^{4} \mathrm{CFUs}$ of a typhoid toxin-deficient isogenic mutant of $S$. Typhi Quailes strain SB6000 (TN). The composite diagnostic end point for typhoid fever was defined as a temperature $\geq 38^{\circ} \mathrm{C}$ persisting for $\geq 12 \mathrm{~h}$ and/or $S$. Typhi bacteremia collected $\geq 72 \mathrm{~h}$ after oral challenge.

Secondary end points were: mode of diagnosis; time to typhoid diagnosis; time to first temperature $\geq 38^{\circ} \mathrm{C}$; fever clearance time; time to bacteremia; duration of bacteremia; and quantitative blood culture (for definitions, see the Life Sciences Reporting Summary). Descriptive end points included: severe adverse events; solicited symptom profiles; proportion of participants meeting the criteria for severe enteric fever; hematological and biochemical measures; plasma cytokine profiles; pattern of bacteremia; and pattern of stool shedding (see Life Sciences Reporting Summary).

Stool samples for culture, blood samples for culture $(10 \mathrm{ml})$, and hematological and biochemical testing were processed by the local hospital's accredited pathology laboratory as described previously ${ }^{7}$

Criteria for severe enteric fever. Severe enteric fever was defined as participants meeting any of the following criteria: oral temperature $>40^{\circ} \mathrm{C}$; systolic blood pressure $<85 \mathrm{mmHg}$; significant lethargy or confusion; gastrointestinal bleeding; gastrointestinal perforation; or any grade 4 laboratory abnormality ${ }^{34}$.

Ex vivo ASC enzyme-linked immune absorbent spot (ELISpot). Ex vivo IgG-, IgA- and IgM-producing ASC responses against $\mathrm{O}$ - and $\mathrm{H}$-antigen were measured at baseline and $24-48 \mathrm{~h}$ after typhoid diagnosis in those meeting the diagnostic criteria as described previously ${ }^{13}$.

Multiscreen filtration ELISpot plates (catalog no. MAHAS4510; Merck Millipore) were coated with S. Typhi O9:LPS, S. Typhi Hd antigen (University of Maryland) and Pan goat anti-human immunoglobulin (catalog no. H17000; Invitrogen) each at a final concentration of $10 \mu \mathrm{g} \mathrm{ml}^{-1}$ in carbonate-bicarbonate buffer and incubated overnight at $4^{\circ} \mathrm{C}$. Plates were blocked with $200 \mu \mathrm{l}$ per well of $\mathrm{R} 10$ medium for $1 \mathrm{~h}$ before use at $37^{\circ} \mathrm{C}, 5 \% \mathrm{CO}_{2}$. Peripheral blood mononuclear cells (PBMCs) were separated using ACCUSPIN tubes (Sigma-Aldrich), counted and resuspended in R10 media. PBMCs at a concentration of $2.5 \times 10^{5}$ were added in duplicate to the ELISpot plate $(100 \mu \mathrm{l}$ per well) and incubated overnight at $37^{\circ} \mathrm{C}, 5 \% \mathrm{CO}_{2}$. Plates were washed four times with PBS- $0.25 \%$ Tween, once with PBS and soaked with PBS for $5 \mathrm{~min}$. Goat anti-human IgG, IgA and IgM secondary antibodies conjugated to alkaline phosphatase (catalog nos. 401442, 401132 and 401902, respectively; Sigma-Aldrich) were diluted to 1:5,000 in PBS/ FBS and incubated for $4 \mathrm{~h}$ at room temperature. After incubation, the plates were washed five times with PBS- $0.25 \%$ Tween and four times with $\mathrm{ds}_{2} \mathrm{O}$. Alkaline phosphatase substrate (catalog no. 170-6432; Bio-Rad) was added at $50 \mu \mathrm{l}$ per well, allowed to develop over approximately $10 \mathrm{~min}$ and stopped with $\mathrm{ds}_{2} \mathrm{O}$ as spots began to develop.

ELISpot plates were read using an automated ELISpot reader (ELR03/ ELR030408215; Autoimmun Diagnostika) and the AID ELISpot software v.5.0. Study- and antigen-specific count settings for spot intensity, size and gradient were applied to the plate counts and manually verified to remove artifacts. Raw counts (spots per $2.5 \times 10^{5} \mathrm{PBMCs}$ ) were averaged across duplicate wells and multiplied by four to give the number of spot-forming units (SFUs) per $10^{6} \mathrm{PBMCs}$.

Fluorospots. Measurements were taken from frozen PBMCs collected at baseline, and on day 14 and 28 post-challenge. Precoated plates (catalog no. FSP-010308-10; Mabtech) were blocked before adding $50 \mu$ l per well toxin peptide pools consisting of 15-mer sequences with 11-amino acid overlaps and covering the sequence of proteins CdtB, PltA and PltB (thinkpeptides). The peptides were dissolved in 100\% DMSO (Sigma-Aldrich) and arranged in three pools. Concentration was adjusted at $0.6 \mathrm{mg} \mathrm{ml}^{-1}$ and used in the fluorospot assay at a final concentration of $3 \mu \mathrm{g} \mathrm{ml}^{-1}$ of each peptide. DMSO and concanavalin A (Sigma-Aldrich) were used as negative and positive controls, respectively. After defrosting and resting for $1 \mathrm{~h}, 50 \mu \mathrm{l}$ per well of PBMCs were added to the peptide wells at a concentration of $4 \times 10^{6}$ cells $\mathrm{ml}^{-1}$ in triplicate and incubated overnight at $37^{\circ} \mathrm{C}, 5 \% \mathrm{CO}_{2}, 95 \%$ humidity. Detection of spots was carried out according to the manufacturer's instructions (Mabtech) and analyzed with the iSpot EliSpot reader (Autoimmun Diagnostika).

Plasma cytokine analysis. Plasma was isolated from heparinized blood by centrifugation. Protease inhibitor was added in a 1:40 dilution before storage at $-80^{\circ} \mathrm{C}$. Longitudinal cytokine quantification was carried out for all 40 challenged participants by the Human Immune Monitoring Center at Stanford University using a 62-plex Luminex system (brain-derived neurotrophic factor, beta-nerve growth factor, CD40 ligand, epidermal growth factor, ENA-78 (CXCL5), eotaxin, fibroblast growth factor 2, granulocyte-colony-stimulating factor, granulocytemacrophage colony-stimulating factor, GRO- $\alpha$ (CXCL1), hepatocyte growth factor, IFN- $\alpha$, IFN- $\beta$, IFN- $\gamma$, IL-1- $\alpha$, IL-1- $\beta$, IL-10, IL-12p40, IL-12p70, IL-13, IL-15, IL-17A, IL-17F, IL-18, IL-1RA, IL-2, IL-21, IL-22, IL-23, IL-27, IL-31, IL-4, IL-5, IL-6, IL-7, IL-8, IL-9, IP-10, leptin, leukemia inhibitory factor, macrophage colonystimulating factor 1 , monocyte chemoattractant protein 1 (MCP-1), MCP-3, MIG, macrophage inflammatory protein-1- $\alpha$ (MIP-1- $\alpha$ ), MIP-1- $\beta$, plasminogen activator inhibitor 1, platelet-derived growth factor subunit B, RANTES, resistin, stem cell factor, stromal cell-derived factor 1, soluble Fas ligand, soluble intercellular adhesion molecule 1 , soluble vascular cell adhesion protein 1 , transforming growth factor- $\alpha$ (TGF- $\alpha$ ), TGF- $\beta$, tumor necrosis factor- $\alpha$ (TNF- $\alpha$ ), TNF- $\beta$, tumor necrosis factor ligand superfamily member 10 , vascular endothelial growth factor A (VEGF-A) and VEGF-D). Samples were run in duplicate and the mean fluorescence intensity (MFI) of duplicates was used for analysis. To minimize plateto-plate variation, samples across time points for each individual were run on the same plates and each plate contained an equal mix of individuals allocated to wildtype or toxin-negative challenge. Control beads (CHEX 1-4) and control sera were used per plate.

The MFI of all samples were examined by principal component analysis to confirm consistency between duplicates and identify outliers. One participant was excluded on this basis. Duplicates were then averaged and the MFI quantile normalized. Significance testing was performed using linear modeling in limma v3.34.9 (ref. ${ }^{35}$ ), incorporating plate, dose and sex as covariates. $P$ values were corrected for multiple testing using the Benjamin-Hochberg correction. Hierarchal clustering was carried out based on Euclidean distance.

Sample size. The sample size was dictated primarily by the number of participants that could be feasibly enrolled within the time frame and budget of the study; therefore, it represents a convenience sample. Assuming typhoid toxin is central to the clinical presentation of acute typhoid fever, it was anticipated that the attack rate following challenge with the TN strain would be reduced compared with the wild-type strain, although the effect size was unknown. Assuming an attack rate of $65 \%$ following wild-type challenge (as observed in previous studies) and $50 \%$ attack rate following TN challenge, and accounting for a $10 \%$ dropout, 20 participants in each group had $95 \%$ CIs for attack rate of $41-85 \%$ in the wild-type group and $27-73 \%$ in the TN group. Twenty participants per arm provided $95 \%$ power to detect an absolute reduction in attack rate of 55\% (65\% with the wildtype strain versus $10 \%$ with the TN strain, corresponding to an $85 \%$ relative risk reduction) and $80 \%$ power to detect an absolute reduction in attack rate of $47 \%$ (65\% with the $S$. Typhi wild-type strain versus $18 \%$ with the $S$. Typhi toxin-negative strain, corresponding to a $72 \%$ relative risk reduction) based on Fisher's exact test with $5 \%$ alpha.

Statistical considerations. Attack rates and 95\% CIs were calculated for each challenge group for the per-protocol population (that is, participants who completed the 14-d challenge period) as the primary end point. All participants were included in the analyses if they were successfully challenged on day 0 and had at least one post-challenge assessment. The difference in attack rate (and other categorical variables) between naive and rechallenge groups was tested using Fisher's exact test. Time-to-event data were summarized using the Kaplan-Meier 
method, with participants censored at day 14. Group comparisons were performed using a log-rank test. Continuous variables were compared using the MannWhitney $U$-test for unpaired samples and the Wilcoxon signed-rank test for paired samples. All statistical tests were two-sided.

Paired samples across time points were compared using the Wilcoxon signedrank test. Comparisons between groups were performed using the Mann-Whitney $U$-test. ELISpot/FluoroSpot data were $\log _{10}$-transformed to approximate a normal distribution; wells with no spots were assigned an arbitrary value of 0.5 , corresponding to half the lower limit of detection. Raw counts were averaged across replicate wells. The number of background spots detected in blank wells were subtracted from the test samples to give the final cell count per sample.

Clinical data were recorded on a web-based database (OpenClinica Enterprise v3.13). Symptom and ELISpot were extracted using Microsoft Excel. Data analysis was performed using R v.3.4.4. Variables were normalized by $z$-score before inclusion in the principal component analysis, which was performed using the FactoMineR package v1.41 (ref. ${ }^{36}$ ).

Approvals. The OVG2016/03 study was sponsored by the University of Oxford (Clinical Trials \& Research Governance). Ethical approvals for the primary protocol, and any study amendments, were obtained from the South CentralOxford A Research Ethics Committee (16/SC/0358). In the UK, legislation governing the deliberate release of genetically modified organisms is currently provided by the Environmental Protection Act 1990, sections 111 and 112 (ref. ${ }^{37}$ ), and the Genetically Modified Organisms (Deliberate Release) Regulations 2002 (ref. ${ }^{38}$ ). Approvals for deliberate release of the genetically modified strain of $S$. Typhi were obtained from the United Kingdom Department for Environment, Food \& Rural Affairs (16/R48/01) (ref. $\left.{ }^{39}\right)$. The study was registered with clinicaltrials.gov (NCT03067961) and was performed according to the provisions of the Declaration of Helsinki (2013) and Good Clinical Practice guidelines. This work is licensed under the Creative Commons Attribution 4.0 International License.

Reporting Summary. Further information on research design is available in the Nature Research Reporting Summary linked to this article.

\section{Data availability}

The datasets generated and/or analyzed during the current study are attached. Any additional data are available from the corresponding author. No participant identifiable information will be disclosed.

The raw sequence reads for the wild-type and TN strains used in the challenge are available under accession nos. ERS3381923 and ERS3381927.

\section{References}

32. Spanò, S. \& Galán, J. E. A Rab32-dependent pathway contributes to Salmonella typhi host restriction. Science 338, 960-963 (2012).

33. Promega. Wizard Genomic DNA Purification Kit (Promega Corporation, 2018); https://ita.promega.com/-/media/files/resources/protocols/technicalmanuals/0/wizard-genomic-dna-purification-kit-protocol.pdf

34. U.S. Food \& Drug Administration. Guidance for Industry. Toxicity Grading Scale for Healthy Adult and Adolescent Volunteers Enrolled in Preventive Vaccine Clinical Trials (U.S. Department of Health and Human Services Food and Drug Administration Center for Biologics Evaluation and Research, 2007); https://www.fda.gov/media/73679/download

35. Ritchie, M. E. et al. limma powers differential expression analyses for RNA-sequencing and microarray studies. Nucleic Acids Res. 43, e47 (2015).

36. Lê, S., Josse, J. \& Husson, F. FactoMineR: An R Package for Multivariate Analysis. J. Stat. Soft. 25, 1-18 (2008).

37. Environmental Protection Act 1990 (The Stationery Office, 1990); https://www.legislation.gov.uk/ukpga/1990/43/pdfs/ukpga_19900043_en.pdf

38. Genetically Modified Organisms (Deliberate Release) Regulations 2002 (Queen's Printer of Acts of Parliament, 2002); http://www.legislation.gov.uk/ uksi/2002/2443/pdfs/uksi_20022443_en.pdf

39. Genetically Modified Organisms: Oxford Vaccine Group (16/R48/01) (GOV.UK, 2016); https://www.gov.uk/government/publications/genetically-modifiedorganisms-oxford-vaccine-group-16r4801 


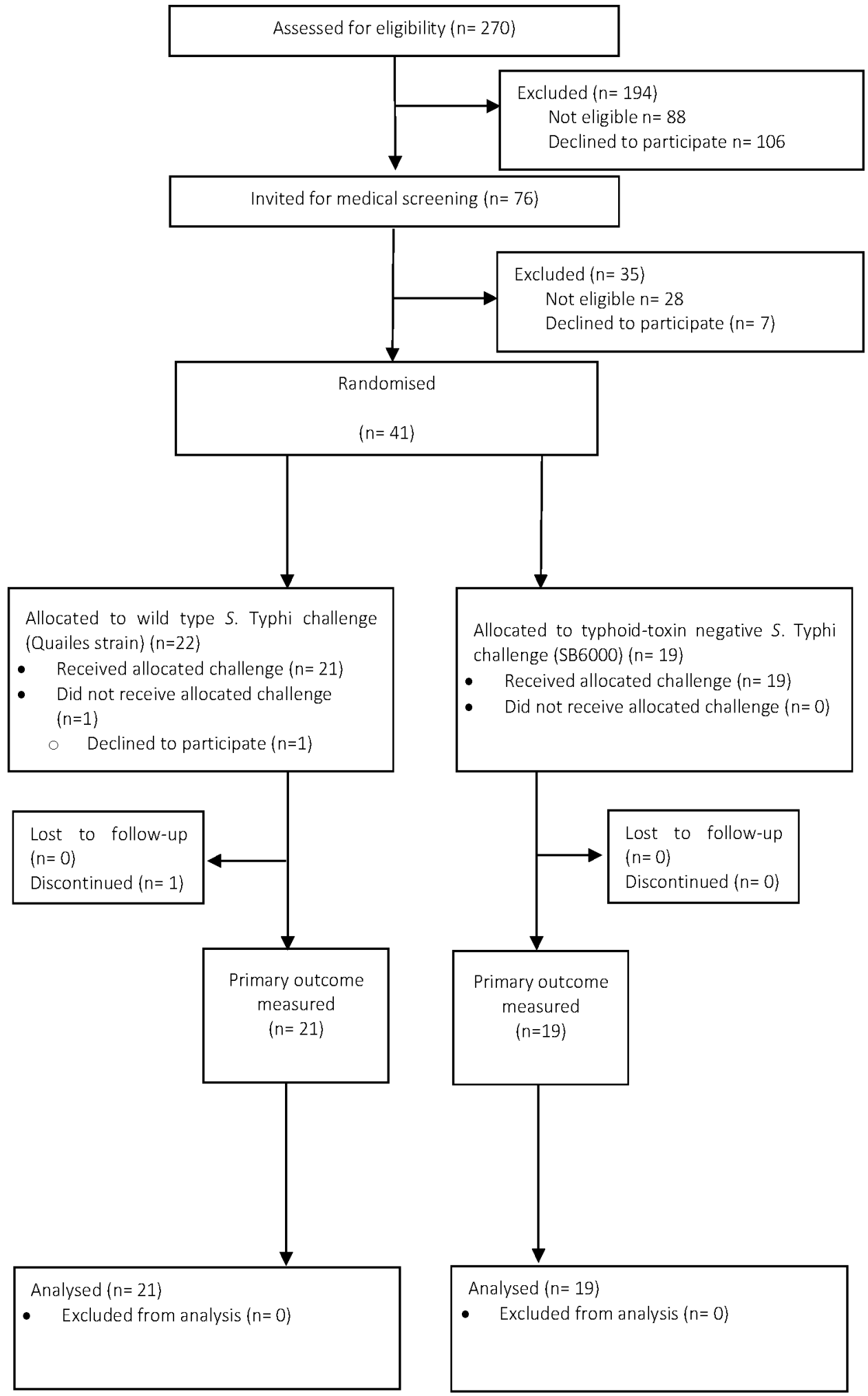

Extended Data Fig. 1 | Trial profile. One participant randomized to wild-type S. Typhi withdrew before challenge and was excluded from all further analyses, leaving 40 participants in the per-protocol analysis. 


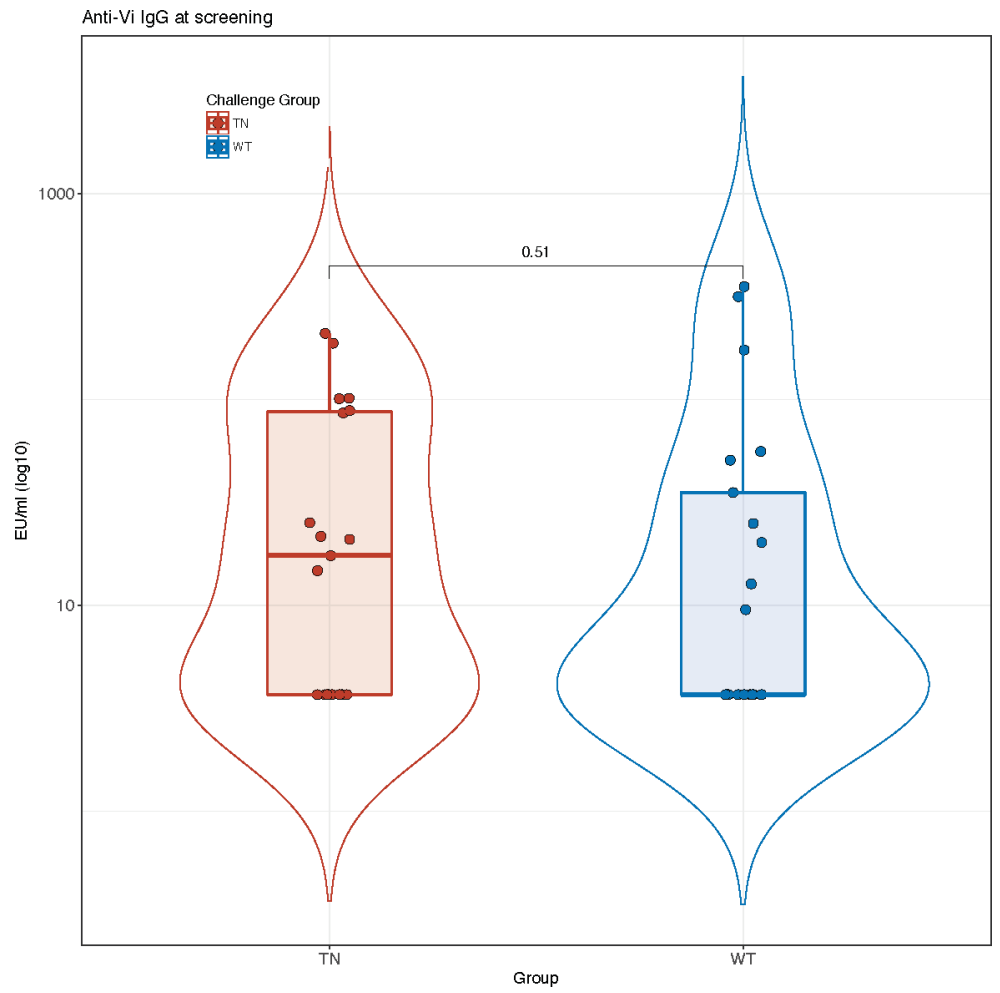

Extended Data Fig. 2 | Baseline anti-Vi IgG. S. Typhi Quailes strain $(n=21)$. Two-sided Mann-Whitney U-test. The box plots represent the median and IQR. The overlaid violin plots illustrate the distribution of the data points and their probability density. Samples below the lower limit of detection of the ELISA (7.4 EU ml-1) were assigned a value equating to half the lower limit of detection (3.7 EU ml-1)14. The box plots display the median and IQR, with the upper whiskers extending to the largest value $\leq 1.5 \times \mathrm{IQR}$ from the 75 th percentile and the lower whiskers extending to the smallest values $\leq 1.5 \times \mathrm{IQR}$ from the 25 th percentile. The overlaid violin plots illustrate the distribution of the data points and their probability density ${ }^{31}$. TN S. Typhi $(n=19)$. 

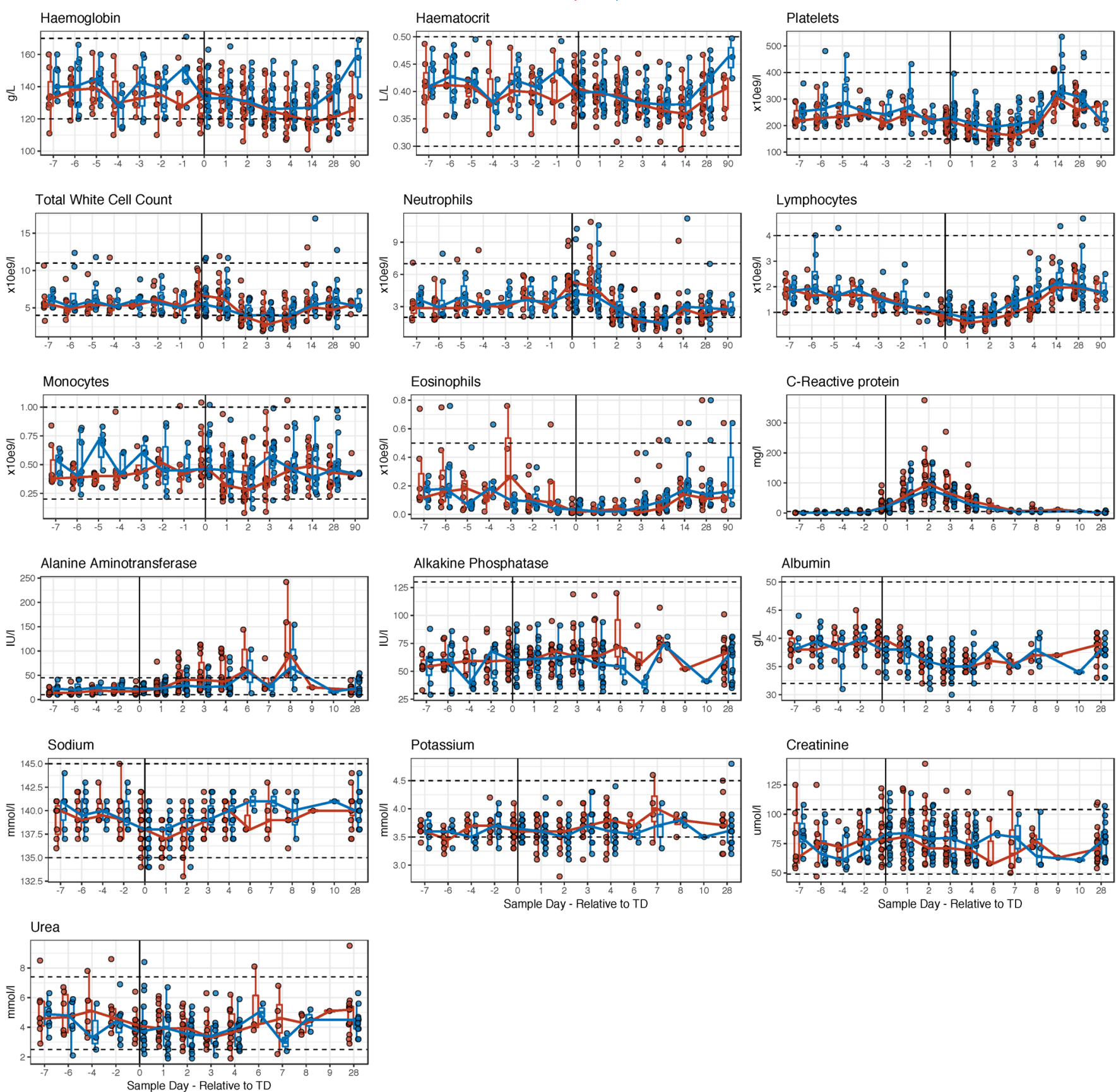

Extended Data Fig. 3 | Hematological and biochemical laboratory measures after challenge. Participants challenged with TN (red) and wild-type (blue) S. Typhi and diagnosed with typhoid fever, $n^{\mathrm{TN}}=15, n^{\text {Wid-type }}=15$. Data are presented relative to the time of typhoid diagnosis (time point $0=$ day of diagnosis). The dot plots corresponds to individual values colored according to challenge group (TN, red; wild-type, blue). The solid, colored lines connect the median values at each time point. The dashed lines represent the upper and lower reference limits for the individual parameters measured. The box plots display the median and IQR, with the upper whiskers extending to the largest value $\leq 1.5 \times I Q R$ from the 75th percentile and the lower whiskers extending to the smallest values $\leq 1.5 \times \mathrm{IQR}$ from the 25 th percentile ${ }^{31}$, and are colored according to challenge group. 


\section{TYGER Study - Principal Component Analysis}

\section{Diagnsosis Status}

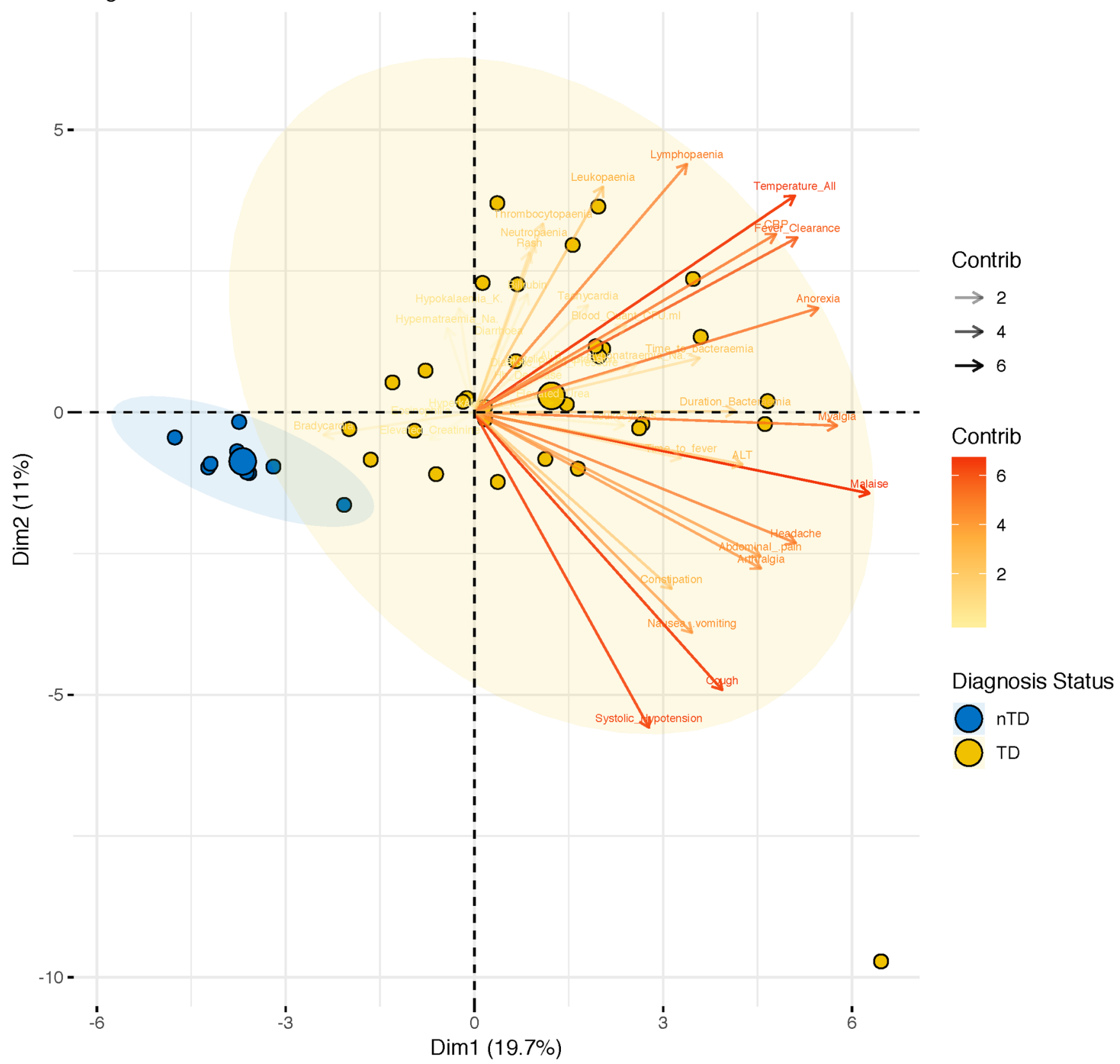

Extended Data Fig. 4 | Principal component analysis (PCA) biplot of disease severity by outcome of challenge. Participants challenged with TN ( $n=19$ ) or wild-type $(n=21) S$. Typhi based on the typhoid severity score. The data points are colored by outcome of challenge (yellow, typhoid diagnosis; blue, no typhoid diagnosis). The ellipses represent the $95 \%$ confidence levels for diagnosis status. The biplot arrows represent the contributions of individual variables to a given principal component, scaled according to their relative contribution ${ }^{36}$. 


\section{TYGER Study PCA}

Challenge Group Allocation

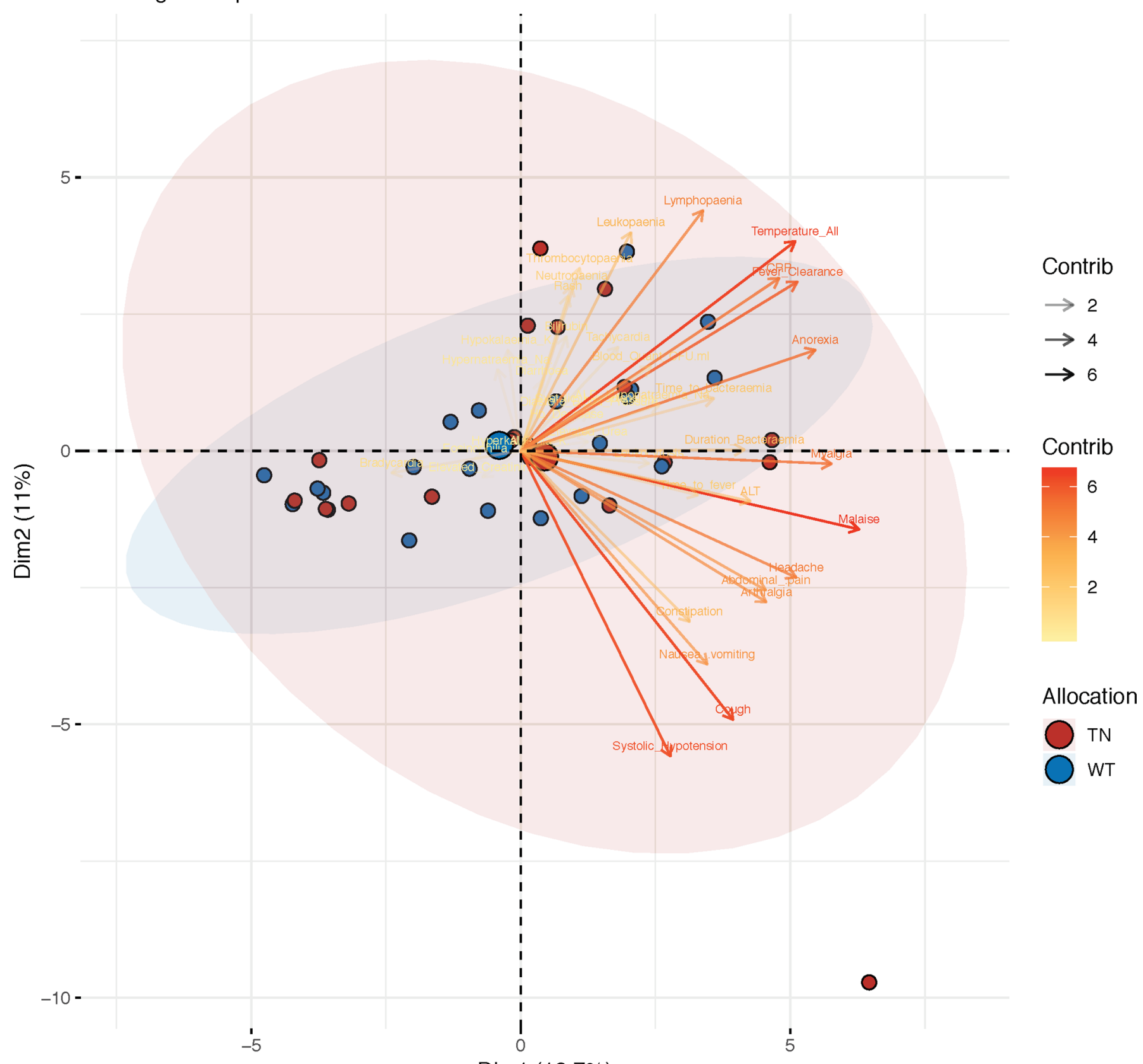

$\operatorname{Dim} 1(19.7 \%)$

Extended Data Fig. 5 | PCA biplot of disease severity by challenge agent. PCA biplot of participants challenged with typhoid fever following challenge with TN $(n=19)$ or wild-type $(n=21)$ S. Typhi based on all clinical, microbiological and laboratory measures. The data points are colored by challenge agent allocation. The ellipses represent the $95 \%$ confidence levels for challenge agent. The biplot arrows represent the contributions of individual variables to a given principal component, scaled according to their relative contribution ${ }^{36}$. 

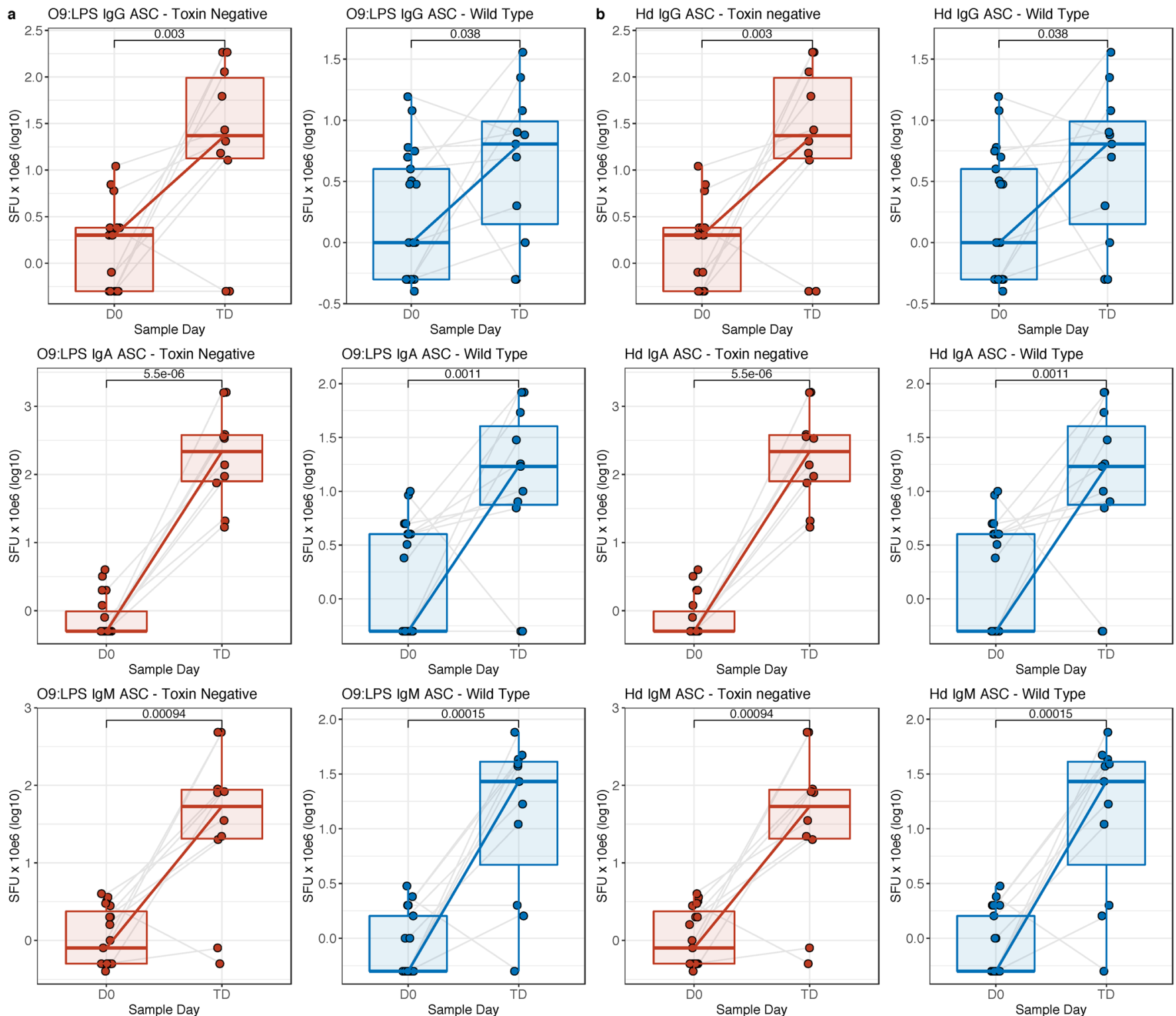

Extended Data Fig. 6 | 09:LPS- and Hd-specific IgG, IgA and IgM ex vivo ASC responses. a, 09:LPS. b, Hd. Participants diagnosed with typhoid, illustrated as $\log _{10}$ SFUs per $10^{6}$ PBMCs. D0, baseline; TD, time of diagnosis (samples processed 24-48 h after initiation of treatment). Two-sided matched-pairs Wilcoxon signed-rank test. $n^{\text {Wid-type }}=11, n^{\text {TN }}=10$. The box plots display the median and IQR, with the upper whiskers extending to the largest value $\leq 1.5 \times$ IQR from the 75 th percentile and the lower whiskers extending to the smallest values $\leq 1.5 \times$ IQR from the 25 th percentile. 


\section{Reporting Summary}

Nature Research wishes to improve the reproducibility of the work that we publish. This form provides structure for consistency and transparency in reporting. For further information on Nature Research policies, see Authors \& Referees and the Editorial Policy Checklist.

\section{Statistics}

For all statistical analyses, confirm that the following items are present in the figure legend, table legend, main text, or Methods section.

n/a Confirmed

$\square$ \The exact sample size $(n)$ for each experimental group/condition, given as a discrete number and unit of measurement

$\square$ \A statement on whether measurements were taken from distinct samples or whether the same sample was measured repeatedly

The statistical test(s) used AND whether they are one- or two-sided

$\triangle$ Only common tests should be described solely by name; describe more complex techniques in the Methods section.

$\square$ \ A description of all covariates tested

$\square$ \ A description of any assumptions or corrections, such as tests of normality and adjustment for multiple comparisons

$\square$ A full description of the statistical parameters including central tendency (e.g. means) or other basic estimates (e.g. regression coefficient)

$\triangle$ AND variation (e.g. standard deviation) or associated estimates of uncertainty (e.g. confidence intervals)

$\square$ For null hypothesis testing, the test statistic (e.g. $F, t, r$ ) with confidence intervals, effect sizes, degrees of freedom and $P$ value noted

$\square$ Give P values as exact values whenever suitable.

Х $\square$ For Bayesian analysis, information on the choice of priors and Markov chain Monte Carlo settings

Х $\square$ For hierarchical and complex designs, identification of the appropriate level for tests and full reporting of outcomes

Х $\square$ Estimates of effect sizes (e.g. Cohen's $d$, Pearson's $r$ ), indicating how they were calculated

Our web collection on statistics for biologists contains articles on many of the points above.

\section{Software and code}

Policy information about availability of computer code

Data collection Clinical data were recorded on a web-based database (OpenClinica Enterprise). Symptom and ELISPOT/Flurospot were extracted using Microsoft Excel. ELISPOT plates were read using an automated ELISPOT reader (AID ELRO3/ELRO30408215) and AID ELISPOT software V5.0. 

was performed using the FactoMineR package 37 with $R$ version 3.4.4.

The randomisation list was generated in STATA version 14.2.

Randomisation was implemented using Sortition ${ }^{\circledast}$ (Oxford University Innovation Ltd \& Nuffield Department of Primary Care, Clinical Trials Unit, University of Oxford).

\section{Sequencing:}

Sequence reads were assembled using HGAP v3 (PMID: 23644548) of the SMRT analysis software v2.3.0 (Available from: https:// github.com/PacificBiosciences/SMRT-Analysis).

The assembly was circularized using Circlator v1.1.3 (PMID: 26714481).

The circularized assembly was polished using the PacBio RS_Resequencing protocol and Quiver v1 of the SMRT analysis software v2.3.0 (Available from: https://github.com/PacificBiosciences/SMRT-Analysis).

Automated annotation, as well as annotation steps on manual assemblies, was performed using PROKKA v1.11 [(PubMed PMID:

24642063) and a genus specific databases from RefSeq (PubMed PMID: 22121212).

SNP/Indel calling

The in-house script uses SMALT v0.7.4 (Available from: https://sourceforge.net/projects/smalt/) to map reads against a selected reference including randomly mapping the repeats and using the GATK indel alignment option. Variation detection was performed using samtools mpileup v0.1.19 and bcftools v0.1.19 to produce a BCF file of all sites and all variant sites.

For manuscripts utilizing custom algorithms or software that are central to the research but not yet described in published literature, software must be made available to editors/reviewers. We strongly encourage code deposition in a community repository (e.g. GitHub). See the Nature Research guidelines for submitting code \& software for further information.

\section{Data}

Policy information about availability of data

All manuscripts must include a data availability statement. This statement should provide the following information, where applicable:

- Accession codes, unique identifiers, or web links for publicly available datasets

- A list of figures that have associated raw data

- A description of any restrictions on data availability

The datasets generated during and/or analysed during the current study are available from the corresponding author. No participant identifiable information will be disclosed.

The raw sequence reads are available under accessions ERS3381923 (sample 1 Oxford w/t), ERS3381924 (sample 2 w/t Pre-GMT), ERS3381925 (sample 3 w/t PostGMT), ERS3381926 (sample 4 k/o Pre-GMT), and ERS3381927 (sample 5 k/o Post-GMT). Manually refined hybrid assemblies as described above are given for the wild type strain (sample 2 w/t Pre-GMT) under accession GCA_901457615 and for the knock-out strain (sample 5 k/o Post-GMT) under accession GCA_901457625.

\section{Field-specific reporting}

Please select the one below that is the best fit for your research. If you are not sure, read the appropriate sections before making your selection. \ Life sciences Behavioural \& social sciences Ecological, evolutionary \& environmental sciences

For a reference copy of the document with all sections, see nature.com/documents/nr-reporting-summary-flat.pdf

\section{Life sciences study design}

All studies must disclose on these points even when the disclosure is negative.

Sample size The number of participants enrolled represents a convenience sample, reflecting the number of participants that could be feasibly enrolled within the time-frame and budget of the study. Assuming that the typhoid toxin is central to the clinical presentation of acute typhoid fever, it was anticipated that the attack rate following challenge with the TN strain would be reduced compared with the WT strain, although the effect size was unknown.

Assuming an attack rate of $65 \%$ following WT challenge and 50\% attack rate following TN strain, and accounting for 10\% drop out, 20 participants in each group gave $95 \%$ confidence intervals for attack rate of $41 \%$ to $85 \%$ in the WT group and $27 \%$ to $73 \%$ in TN group. Twenty participants per arm provided $95 \%$ power to detect a relative risk reduction in attack rate of $85 \%$ (65\% with WT strain versus $10 \%$ with TN strains) and $80 \%$ power to detect a relative reduction in attack rate of $72 \%$ ( $65 \%$ with S. Typhi wild type strain versus $18 \%$ with S. Typhi toxinnegative) based on Fisher's Exact test with 5\% alpha

Data exclusions One participant randomized to wild-type S. Typhi declined to participate after enrolment, but prior to challenge, and was excluded from all further analyses.

Replication Participants acted as biological replicates within study groups.

Permission for deliberate release of the genetically modified toxin-negative strain of $\mathrm{S}$. Typhi was given for a limited number of participants $(n=20)$ and precluded replication in an independent cohort.

Randomization Participants were randomized 1:1 to challenge with either wild-type strain Salmonella Typhi (WT) or toxin-negative strain Salmonella Typhi (TN) in varying block sizes. 
Anti-Vi IgG was measured at screening using a commercial ELISA kit (VaccZyme, The Binding Site Ltd, Birmingham, UK) according to the manufacturer's instructions. Randomization was stratified by anti-Vi lgG (low $<7.4 \mathrm{EU} / \mathrm{ML}$ or high $=>7.4 \mathrm{EU} / \mathrm{ml}$. The exception was a sentinel group of two participants who were randomized 1:1 to receive the WT strain or TN knock-out strain, using a block size of two.

Randomization was performed at the pre-challenge visit (Day -7), one week prior to challenge. We generated a randomisation list in STATA version 14.2, which was implemented in the computerised randomisation software Sortition (Nuffield Department of Primary Care, Clinical Trials Unit, University of Oxford), which matched a masked allocation group to each participant.

\section{Reporting for specific materials, systems and methods}

We require information from authors about some types of materials, experimental systems and methods used in many studies. Here, indicate whether each material, system or method listed is relevant to your study. If you are not sure if a list item applies to your research, read the appropriate section before selecting a response.

\begin{tabular}{l|l} 
Materials \& experimental sys \\
\hline $\mathrm{n} / \mathrm{a}$ & Involved in the study \\
$\square$ & $\bigotimes$ Antibodies \\
$\square$ & $\square$ Eukaryotic cell lines \\
$\square$ & $\bigotimes$ Animals and other organisms \\
$\square$ & $\bigotimes$ Clinical data
\end{tabular}

\begin{tabular}{l|l}
\multicolumn{2}{l}{ Methods } \\
\hline n/a & Involved in the study \\
$\square$ & $\square$ ChIP-seq \\
$\square$ & $\square$ Flow cytometry \\
$\square$ & $\square$ MRI-based neuroimaging
\end{tabular}

Antibodies

Antibodies used

Antibodies:

Manufacturer: Merck Millipore

Catalog/lot number: Goat anti-human IgG -Chain specific conjugated to alkaline phosphatase $401442-1 \mathrm{ml} / 2689825$

Goat anti-human IgA alpha-Chain specific conjugated to alkaline phosphatase 401132/D00165538.

Used at a 1:5000 dilution.

Flurospot detection antibodies, using Mabtech kit FSP-010308-10 (batch 3).

Manufacturer: Mabtech

Anti IFNg monoclonal antibody, clone 7-B6-1-BAM, batch 1, 1/200 dilution

Anti IL17A monoclonal antibody biotinylated, clone MT504, batch 7, 1/250 dilution

Fluorophore conjugates:

anti BAM-490, batch 3, 1/200 dilution; SA-550, batch 7, 1/200 dilution; anti-WASP-641, batch 7, 1/200 dilution

Validation

Immunoaffinity purified goat polyclonal anti-human IgA and anti-human IgG antibody conjugated to alkaline phosphatase, validated by manufacturer Merck Millipore.

(Manufacturers information available at http://www.merckmillipore.com/GB/en/product/Goat-Anti-Human-IgG-Chain-SpecificAlkaline-Phosphatase-Conjugate,EMD_BIO-401442\#anchor_PDS)

Antibodies used in flurospot assay validated by manufacturer Mabtech

(Manufacturers information available at https://www.mabtech.com/products/anti-human-ifn-gamma-antibody-7-b6-1-

biotinylated-3420-6)

Antibodies used in ex-vivo ELISPOT assays as outlined in https://www.ncbi.nlm.nih.gov/pubmed/18032593 and https:// www.ncbi.nlm.nih.gov/pubmed/27533046

\section{Eukaryotic cell lines}

Policy information about cell lines

Cell line source(s)

Henle-407 intestinal epithelial cells from the Roy Curtiss laboratory collection.

Authentication

The cells were frequently checked for their morphological features, growth speed and functionalities, but were not 


\section{Authentication}

\section{Mycoplasma contamination}

Commonly misidentified lines (See ICLAC register) authenticated by short tandem repeat (STR) profiling.

All cell lines tested negative for mycoplasma contamination.

No commonly misidentified cell lines were used.

\section{Animals and other organisms}

Policy information about studies involving animals; ARRIVE guidelines recommended for reporting animal research

Laboratory animals $\quad \mathrm{CmaH}$-/- bloc3-/- mice (Figure 1).

Age: 7 -10 week old

Sex: male and females, randomly assigned to the different groups

Wild animals

The study did not involve wild animals.

Field-collected samples

The study did not involve field collected specimens.

Ethics oversight

All animal experiments were conducted according to protocols approved by Yale University's Institutional Animal Care and Use Committee.

Note that full information on the approval of the study protocol must also be provided in the manuscript.

\section{Human research participants}

\section{Policy information about studies involving human research participants}

Population characteristics Healthy adults aged $18-60$ years

Key exclusion criteria included significant medical, surgical or psychiatric history, gallbladder disease and high-risk occupations as defined by Public Health England guidelines. A full description of inclusion and exclusion criteria is provided in the attached study protocol and below.

\section{Study eligibility}

Male or female participants aged 18-60 years inclusive who were in good health (as determined by a study doctor, medical investigation and review of medical history provided by their General Practitioner) and who were able to provide written informed consent were eligible for inclusion in this study.

\section{Inclusion Criteria}

Participants must satisfy all of the following criteria to be considered eligible for the study:

- Agree to give informed consent for participation in the study.

- Aged between 18 and 60 years inclusive at time of challenge.

- In good health as determined by medical history, physical examination and clinical judgment of the study team.

- Agree (in the study team's opinion) to comply with all study requirements, including capacity to adhere to good personal hygiene and infection control precautions.

- Agree to allow his or her General Practitioner (and/or Consultant if appropriate), to be notified of participation in the study.

- Agree to allow study staff to contact his or her GP to access the participant's vaccination records and summary of medical history.

- Agree to allow Public Health England to be informed of their participation in the study.

- Agree to give his or her close contacts written information informing them of the participant's involvement in the study and offer them voluntary screening for $\mathrm{S}$. Typhi carriage.

- Agree to have 24-hour contact with study staff during the four weeks post challenge and to be able to ensure that they are contactable by mobile phone for the duration of the challenge period until antibiotic completion.

- Agree to allow the study team to hold the name and 24-hour contact number of a close friend, relative or housemate who will be kept informed of the study participant's whereabouts for the duration of the challenge period (from the time of challenge until completion of antibiotic course). This person will be contacted if study staff are unable to contact the participant.

- Have internet access to allow completion of the e-diary and real-time safety monitoring.

- Agree to avoid antipyretic/anti-inflammatory treatment from the time of challenge (Day 0) until advised by a study doctor or until 14 days after challenge.

- Agree to refrain from donating blood for the duration of the study.

- Agree to provide their National Insurance/Passport number for the purposes of TOPS registration and bank account details for payment of reimbursement expenses.

\section{Exclusion Criteria}

Participants were not enrolled if any of the following applied:

- History of significant organ/system disease that could interfere with trial conduct or completion. Including, for example, but not restricted to:

- Cardiovascular disease

o Respiratory disease

o Haematological disease

o Endocrine disorders

o Renal or bladder disease, including history of renal calculi 
o Biliary tract disease, including biliary colic, asymptomatic gallstones or previous cholecystectomy

o Gastro-intestinal disease including requirement for antacids, H2-receptor antagonists, proton pump inhibitors or laxatives o Neurological disease

o Metabolic disease

o Autoimmune disease

o Psychiatric illness requiring hospitalisation or known or suspected drug and/or alcohol misuse (alcohol misuse defined as an intake exceeding 42 units per week)

o Infectious disease

- Have any known or suspected impairment of immune function, alteration of immune function, or prior immune exposure that may alter immune function to typhoid resulting from, for example:

o Congenital or acquired immunodeficiency, including IgA deficiency

o Human Immunodeficiency Virus infection or symptoms/signs suggestive of an HIV-associated condition

o Receipt of immunosuppressive therapy such as anti-cancer chemotherapy or radiation therapy within the preceding 12 months or long-term systemic corticosteroid therapy.

o Receipt of immunoglobulin or any blood product transfusion within 3 months of study start.

o History of cancer (except squamous cell or basal cell carcinoma of the skin and cervical carcinoma in situ).

- Moderate or severe depression or anxiety as classified by the Hospital Anxiety and Depression Score at screening or challenge that is deemed clinically significant by the study doctors .

- Weight less than $50 \mathrm{~kg}$.

- Presence of implants or prosthesis.

- Anyone taking long-term medication (e.g. analgesia, anti-inflammatories or antibiotics) that may affect symptom reporting or interpretation of the study results.

- Contraindication to fluoroquinolone or macrolide antibiotics (e.g. ciprofloxacin or azithromycin).

- Female participants who are pregnant, lactating or who are unwilling to ensure that they or their partner use effective contraception 30 days prior to challenge and until three negative stool samples have been obtained after completion of antibiotic treatment.

- Full-time, part-time or voluntary occupations involving:

o Clinical or social work with direct contact with young children (defined as those attending pre-school groups or nursery or aged under 2 years), or

o Clinical or social work with direct contact with highly susceptible patients or persons in whom typhoid infection would have particularly serious consequences (unless willing to avoid work until demonstrated not to be infected with S. Typhi in accordance with guidance from Public Health England and willing to allow study staff to inform their employer).

- Full time, part time or voluntary occupations involving:

o Commercial food handling (involving preparing or serving unwrapped foods not subjected to further heating)

- Close household contact with:

o Young children (defined as those attending pre-school groups, nursery or those aged less than 2 years)

o Individuals who are immunocompromised.

- Scheduled elective surgery or other procedures requiring general anaesthesia during the study period.

- Participants who have participated in another research study involving an investigational product that might affect risk of typhoid infection or compromise the integrity of the study within the 30 days prior to enrolment (e.g. significant volumes of blood already taken in previous study)

- Detection of any abnormal results from screening investigations (at the clinical discretion of the study team).

- Inability to comply with any of the study requirements (at the discretion of the study staff and the participant's General Practitioner).

- Any other social, psychological or health issues which, in the opinion of the study staff, may

o put the participant or their contacts at risk because of participation in the study,

o adversely affect the interpretation of the primary endpoint data,

o impair the participant's ability to participate in the study.

- Prior vaccination with an oral typhoid vaccines (e.g. Ty21a) or other investigational typhoid vaccine (e.g. Vi-conjugate vaccine, M01ZH09)

- Prior vaccination with a Vi-polysaccharide typhoid vaccine administered within 5 years from the time of screening.

- Prior vaccination with a Vi-polysaccharide typhoid vaccine administered more than 5 years from the time of screening AND detectable Vi-antibody titre at screening (defined as $\operatorname{lgG} \geq 7.4 \mathrm{U} / \mathrm{ml}$ measured using the VaccZyme Salmonella Typhi Vi IgG kit, Binding Site $\left.{ }^{\circledR} \mathrm{UK}\right)$.

- Having been resident in an enteric fever endemic country for 6 months or more.

- Have previously been diagnosed with laboratory-confirmed typhoid or paratyphoid infection or been given a diagnosis compatible with enteric fever.

- Have participated in previous typhoid or paratyphoid challenge studies (with ingestion of challenge agent).

- Have a prolonged corrected QT interval (>450 milliseconds) on ECG screening.

Several strategies were employed to recruit participants, including:

-NHS database: Potential study participants will be identified via databases such the National Health Applications and Infrastructure Services (NHAIS) who hold the central NHS patient database (Open Exeter) or their equivalent.

-Poster advertising: Display of posters advertising the study throughout local hospitals and doctor's surgeries, tertiary education institutions and other public places with the permission of the owner/ proprietor.

-Direct mail-out

-E-mail communication: We will contact representatives of local tertiary education establishments and local employers and ask them to circulate posters and information, and to circulate a link to study information on the OVG website by email. 
-Oxford Vaccine Centre (OVC) database for healthy volunteers

-Media advertising: Local media, newspaper, radio, website and social media advertisement placed in locations relevant for the target age group with brief details of the study and contact details for further information

-Website advertising: Description of the study and copy of information booklet on the Oxford Vaccine Group website.

Exhibitions: Advertising material and/or persons providing information relating to the study will exhibit using stalls or stands at exhibitions and/or fairs, such as University Fairs

Royal Mail Leaflet: Royal Mail door-to-door service with delivery of invitation letters in OVG envelopes to every household within certain postcode areas.

Potential participants who were interested in study participation contacted the study site by telephone, email, by out website online registration with self-screening questions or paper reply slip for further information. Once an expression of interest was received, an information booklet was be sent via mail or email to the potential participants to read at their leisure. Participants were also be directed to the Oxford Vaccine Group website, where the information booklet was available. If participants were willing to proceed they were invited for a screening and consent visit, where a member of the clinical research team at the Oxford Vaccine Group assessed their eligibility. We also took consent for clinical staff to access electronic patient records (EPR) to assess eligibility.

We acknowledge the potential self-selection bias in such human-challenge studies. We contend that $t$ any potential biases are mitigated by standardized procedures and consistent inclusion/exclusion criteria. These limitations are discussed within the manuscript.

Ethics oversight

The OVG2016/03 study was sponsored by the University of Oxford (Clinical Trials \& Research Governance). Ethical approvals for the primary protocol, and any study amendments, were obtained from the South-Central Oxford A research ethics committee (16/SC/0358). In the UK, legislation governing the deliberate release of genetically modified organisms is currently provided by the Environmental Protection Act 1990 section 111 and 11242, and the Genetically Modified Organisms (Deliberate Release) Regulations 200243. Approvals for deliberate release of the genetically modified strain of S. Typhi were obtained from the United Kingdom Department for Environment, Food \& Rural Affairs (16/R48/01)44

Note that full information on the approval of the study protocol must also be provided in the manuscript.

\section{Clinical data}

Policy information about clinical studies

All manuscripts should comply with the ICMJE guidelines for publication of clinical research and a completed CONSORT checklist must be included with all submissions.

Clinical trial registration

Study protocol

Data collection

Outcomes
Clinicaltrials.gov NCT03067961

Submitted alongside manuscript

Centre for Clinical Vaccinology \& Tropical Medicine, The Churchill Hospital, Oxford, United Kingdom OX3 7LE

10th April 2017 and 1st August 2017

Primary Outcome Measure

The primary outcome was the attack rate post challenge, defined as the proportion of participants meeting the composite diagnostic endpoint for typhoid fever during the challenge period.

The composite diagnostic endpoint for typhoid fever was defined as a temperature of $38 \mathrm{C}$ persisting for $>12 \mathrm{hrs}$ and/or S. Typhi bacteraemia collected $>72$ hours after oral challenge

Secondary clinical and microbiological outcome variables are listed by below

- Time to diagnosis - Time from date/time of challenge to date/time of first temperature $>38 \mathrm{C}$ that subsequently lasted for $>12$ hours OR the date/time of first positive blood culture collection (whichever occurs earliest).

- Time to first blood culture positive for S. Typhi- Time from date/time of challenge to date/time of blood culture collection. - Time to clinical diagnosis (fever $>38 \mathrm{C}$ lasting $>12$ hours) - Time from date/time of challenge to date/time of first recorded temperature $>38 \mathrm{C}$ which subsequently lasted 12 hours.

- Mode of diagnosis - The proportion of participants diagnosed with typhoid/paratyphoid fever based upon either clinical criteria (persistent fever $>38 \mathrm{C}$ for $>12$ hours) OR microbiological criteria (blood culture positive for S. Typhi collected $>72$ hours from diagnosis)

- Detailed mode of diagnosis - Proportion of participants diagnosed with typhoid/paratyphoid fever based upon either of the

following specific diagnostic criteria:

Temperature $>38 \mathrm{C}$ preceding positive blood culture;

o Temperature $>38 \mathrm{C}$ without positive blood culture;

o S. Typhi bacteraemia preceding temperature $>38 \mathrm{C}$;

o S. Typhi bacteraemia without temperature $>38 \mathrm{C}$.

- Time to first fever - Time from date/time of challenge to date/time of first recorded temperature $>38 \mathrm{C}$

- Fever clearance time - Time from initiation of antibiotics or start of fever (whichever was later) to first recorded temperature

$<380 \mathrm{C}$ persisting for at least 48hours. Only diagnosed participants with fever were included in the analysis. 
- Symptom severity - Severity of symptoms in each challenge group were assessed by:

o The proportion of participants with maximum symptom severity score graded as mild, moderate or severe following challenge. o The proportion of participants meeting the criteria for severe enteric fever.

o Individual enteric fever severity scores calculated by summing numerical values assigned to the severity of individual solicited symptoms, clinical observations (heart rate, systolic blood pressure, diastolic blood pressure and temperature) and laboratory measurements between Day 0 to Day 21 ( $0=$ not present; $1=$ mild; $2=$ moderate; $3=$ severe; 4 = Hospitalisation.

o Duration of bacteraemia - Time (Hours/Days) from collection of first positive blood culture until date/time of the last positive blood culture.

o Bacteraemia clearance time - Time (Hours/Days) from collection of first positive blood culture until date/time of the first negative blood culture remaining persistently negative. Participants with missing data (e.g. no negative blood cultures after commencing antibiotics) were censored in the analysis at the time point of the last culture taken.

o Stool shedding - Daily stool culture(s) positive for S. Typhi for 14 days post-challenge.

- Quantitative blood culture - Concentration of bacteria in $10 \mathrm{ml}$ blood taken at the time of diagnosis using the Wampole ${ }^{\mathrm{Tm}}$ Isostat ${ }^{\circledR}$ Isolator system (Colony forming units $/ \mathrm{ml}$ ). For values below the lower limit of detection $(0.1 \mathrm{CFU} / \mathrm{ml}$ ), a value of 0.05 $\mathrm{CFU} / \mathrm{ml}$ was assigned.

- Haematological and biochemical end-points - The following haematological parameters were measured from time of challenge to Day 28 and/or Day 90.

o Total Haemoglobin (g/L)

o Haemoglobin change from baseline ( $\mathrm{Hb}$ g/l DO - Hb g/l D14)

o Total White Cell Count (x109/I)

o Platelet counts (x109/I)

o Neutrophil count (x109/I)

o Lymphocyte count (x109/I)

o Monocyte count (x109/I)

o Eosinophil count (x109/l)

o Monocyte/Lymphocyte ratio

o Urea \& Electrolytes ( $\mathrm{Na}, \mathrm{K}+$, Urea, Creatinine -mmol/l)

o C-reactive protein $(\mathrm{mg} / \mathrm{l})$

o Liver function tests (Bilirubin [umol/I], aspartate transaminase (AST IU/I), alkaline phosphatase (ALP IU/I), alanine transaminase (ALTIU/I), Albumin (g/L)

- Safety outcome measures - Adverse events, adverse events of special interest, SAE's and SUSARs according to each study group. 